\title{
POLARIZATION PHENOMENA BY DEUTERON FRAGMENTATION INTO PIONS
}

\author{
A.Yu. Illarionov, A.G. Litvinenko and G.I. Lykasov \\ Joint Institute for Nuclear Research, \\ 141980 Dubna, Moscow Region, Russia
}

February 8, 2020

\begin{abstract}
The fragmentation of deuterons into pions emitted forward in the kinematic region forbidden for free nucleon-nucleon collisions is analyzed. The inclusive relativistic invariant spectrum of pions and the tensor analyzing power $T_{20}$ are investigated within the framework of an impulse approximation using different kinds of the deuteron wave function. The influence of $P$-wave inclusion in the deuteron wave function is studied, too. The invariant spectrum is shown to be more sensitive to the amplitude of the $N N \rightarrow \pi X$ process than the tensor analyzing power $T_{20}$. Our results are compared with the experimental data and other calculations performed within both the non-relativistic and relativistic approaches.
\end{abstract}

Key-words: cumulative processes, polarization observables, deuteron fragmentation, Bethe-Salpeter amplitude, deuteron wave function, small components, non-nucleon components, quark degrees of freedom, minimal relativization procedure.

\section{INTRODUCTION}

One of the main sources of information about the deuteron structure at small distances are the reactions of hadrons production by proton-deuteron and deuteron-deuteron collisions in the kinematic region forbidden for the free nucleon-nucleon interaction [1, 2], the so-called cumulative processes. This kinematic region corresponds to the values of the light-cone variable $x=2\left(E^{\prime}+p^{\prime}\right) /\left(E_{D}+p_{D}\right) \geq 1$, where $E^{\prime}, E_{D}$ and $p^{\prime}, p_{D}$ are the energies and momenta of the final hadron and deuteron, respectively. The nucleon momentum distributions in the deuteron, extracted from the reaction $D p \rightarrow p X$ at forward proton emission and $e D$-inelastic scattering [3] actually coincide with each other (see, for example, [4]). So, one can conclude that hadron and lepton probes result in the same information about the deuteron structure. The so-called Paris [5] and Reid [6] deuteron wave functions (DWF) reproduce rather well the experimental data on the $D p \rightarrow p X$ reaction for internal momenta $k=\sqrt{m^{2} /(4 x(1-x))-m^{2}}$ up to $0.25 \mathrm{GeV} / \mathrm{c}$ within the framework of an impulse approximation (IA) [4]). The inclusion of corrections to IA related to secondary interactions allows one to describe the experimental data on the deuteron fragmentation $D p \rightarrow p X$ at $k>0.25 \mathrm{GeV} / \mathrm{c}$ [7].

The investigation of polarization phenomena by deuteron fragmentation at middle and high energies in the kinematic region forbidden for hadrons emission by free $N-N$ scattering has recently become very topical. Cumulative proton production in the collision of polarized deuterons with the target results in information about the deuteron spin structure at small inter-nuclear distances. This can be seen from the experimental and theoretical study of deuteron fragmentation into protons at a zero angle [8, 9, 7]. The theoretical analysis of this reaction has shown that the tensor analyzing power $T_{20}$ and polarization transfer coefficient $\kappa$ are more sensitive to the used deuteron wave function (DWF), particular to the reaction mechanism, than the inclusive spectrum [7]. At the present time, not one DWF relativistic form can describe $T_{20}$ at $x \geq 1.7$ measured by $D p \rightarrow p X$ stripping. On the other hand, the inclusion of the reaction mechanism: namely the impulse approximation and the secondary interaction of produced hadrons can describe both the inclusive spectrum and $T_{20}$ at $x \leq 1.7$ using only the nucleon degrees of freedom [7]. One of the causes, to explain this phenomenon can be the fact that the deuteron structure at a high $(>0.20 \mathrm{GeV} / \mathrm{c})$ internal momentum (short 
inter-nuclear distances $<1 \mathrm{fm}$ ) is determined by non-nucleon degrees of freedom. The inclusion of non-nucleon degrees of freedom, (it can be either the six-quark state or the composition of $\Delta \Delta$, $N N^{\star}, N N \pi$ and other states in the deuteron) allowed one to describe the experimental data on the inclusive proton spectrum at $x \geq 1.7$ [7]. A number of papers were dedicated to a theoretical analysis of the deuteron stripping to protons, see for example Refs. in [2, 7]. However, to date there has been no unified theoretical description of $T_{20}$ on the whole kinematic region of protons emitted forward by the $D p \rightarrow p X$ striping.

If we try to study the manifestation of non-nucleon degrees of freedom, it is natural to investigate the cumulative production of different hadrons having different quark contents. Interesting experimental data on $T_{20}$ in the reaction $D p \rightarrow \pi X$ where the pion is emitted forward have been published recently [10]. They show very small, approximately constant, value of the tensor analyzing power $T_{20}$ for the deuteron fragmentation of into pions $D p \rightarrow \pi X$ at $x \geq 1$. The mechanism of this reaction is mainly an impulse approximation as the secondary interaction or the final state interaction (FSI) is very small and can be neglected [11]. Quite large yield of high momentum pions produced by $p-D$ and $p-A$ collisions in the kinematical region forbidden for free $N-N$ scattering was explained by both few-nucleon correlation models [2] and [12] or a multi-quark bags one [13, [14]. However, the polarization phenomena in deuteron fragmentation into pions were outside of these models.

In this paper, we present a relativistic invariant analysis of the deuteron tensor analyzing power $T_{20}$ and unpolarized pion spectrum in the backward inclusive $p+D \rightarrow \pi\left(180^{0}\right)+X$ reaction (in deuteron rest frame). The main goal is to describe this reaction in a consistent relativistic approach using the nucleon model of the deuteron. A fully covariant expression for all quantities is obtained within the Bethe-Salpeter (BS) formalism. This way results in general conclusions of the process amplitude which can not be seen in the non-relativistic approach. On the other hand, the nonrelativistic limit will be recovered, and some links to non-relativistic corrections can be found. This analysis of the deuteron models can be very important to search for nuclear quark phenomena.

\section{RELATIVISTIC IMPULSE APPROXIMATION}

Let us consider the inclusive reaction $\boldsymbol{D}+p \rightarrow \pi\left(0^{0}\right)+X$ within the framework of the impulse approximation.

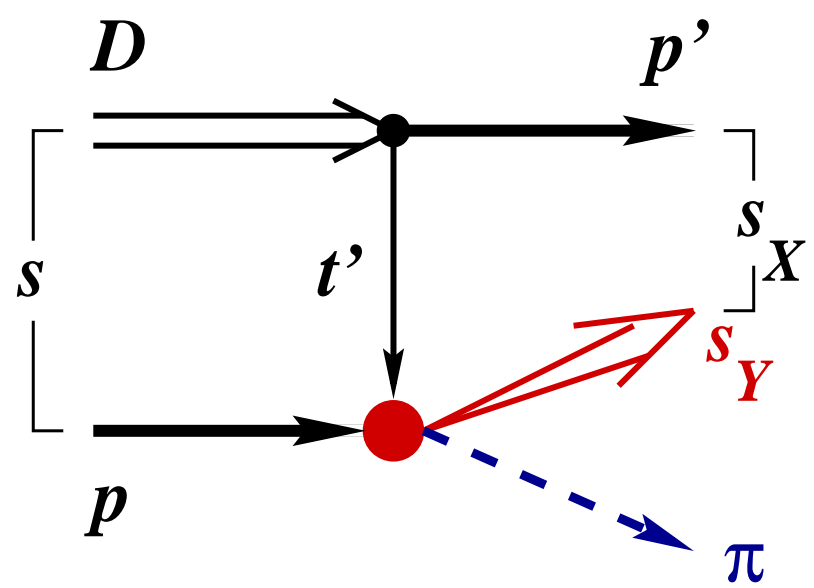

The amplitude of this process $\mathcal{T}_{p D}^{\pi}$ can be written in the following relativistic invariant form:

$$
\mathcal{T}_{p D}^{\pi} \equiv \mathcal{T}(D p \rightarrow \pi X)=\left(\overline{\mathcal{U}}_{Y} \Gamma_{N N}\right)_{\alpha \beta} \bar{u}_{\gamma}^{\left(\sigma_{p^{\prime}}\right)}\left(p^{\prime}\right)\left(\frac{\widehat{n}+m}{n^{2}-m^{2}}\right)_{\beta \delta} u_{\alpha}^{\left(\sigma_{p}\right)}(p)\left(\Gamma_{\mu}(D, q) \mathcal{C}\right)_{\delta \gamma} \xi_{M}^{\mu}(D)
$$

where $\left(\overline{\mathcal{U}}_{Y} \Gamma_{N N}\right)$ is the truncated $N N \rightarrow \pi Y$ vertex; $\alpha, \beta, \gamma$ and $\delta$ are the Dirac indices (with summation over twofold indices); $\mu$ is a Lorentz index; $\mathcal{C}=i \gamma_{2} \gamma_{0}$ is a charge conjugation Dirac matrix and $M$ is the deuteron spin projection. Here, the deuteron vertex $\left(\Gamma_{\mu}(D, q) \mathcal{C}\right)$ satisfies the $B S$ equation and depends on the relative momentum $q=\left(n-p^{\prime}\right) / 2$ and total momentum $D=n+p^{\prime}$ of deuteron, 
$\xi_{M}^{\mu}(D)$ is the four-vector of the deuteron polarization. It satisfies the following equations:

$$
\begin{gathered}
\xi^{\mu M}(D) D_{\mu}=0, \quad \xi^{\mu M}(D) \xi_{\mu M^{\prime}}(D)=-\delta_{M^{\prime}}^{M} \\
\sum_{M}\left(\xi_{\mu M}(D)\right)^{*} \xi_{\nu M}(D)=-g_{\mu \nu}+\frac{D_{\mu} D_{\nu}}{M^{2}}
\end{gathered}
$$

Squaring this amplitude, one can write the relativistic invariant inclusive pion spectrum of the reaction $D p \rightarrow \pi X$ in the following form:

$$
\rho_{p D}^{\pi}=\varepsilon_{\pi} \frac{d \sigma}{d^{3} p_{\pi}}=\frac{1}{(2 \pi)^{3}} \int \frac{\sqrt{\lambda(p, n)}}{\sqrt{\lambda(p, D)}} \rho_{\mu \nu}(D)\left[\rho_{p N}^{\pi} \cdot \Phi^{\mu \nu}(D, q)\right] \frac{m^{2} d^{3} p^{\prime}}{E^{\prime}}
$$

where $\lambda\left(p_{1}, p_{2}\right) \equiv\left(p_{1} p_{2}\right)^{2}-m_{1}^{2} m_{2}^{2}=\lambda\left(s_{12}, m_{1}^{2}, m_{2}^{2}\right) / 4$ is the flux factor; $p, n$ are the four-momenta of the proton-target and intra-deuteron nucleon, respectively; $\rho_{p N}^{\pi} \equiv \varepsilon_{\pi} \cdot d \sigma / d^{3} p_{\pi}$ is the relativistic invariant inclusive spectrum of pions produced by interacting the intra-deuteron nucleon with the proton-target. In the general case, this spectrum can be written as a three-variable function $\rho_{p N}^{\pi}=$ $\rho\left(x_{f}, \pi_{\perp}, s_{N N}\right)$. Feynman's variable, $x_{f}$, is defined as $x_{f}=2 \pi_{\|} / \sqrt{s_{N N}}$, where $\pi$ is the pion momentum in the center of mass of two interacting nucleons and $s_{N N}=(p+N)^{2}$.

$\rho_{\mu \nu}(D)$ is the density matrix of the deuteron [15]:

$$
\begin{aligned}
& \rho_{\mu \nu}(D)=\left(\xi_{\mu M}(D)\right)^{*} \xi_{\nu M}(D)=\frac{1}{3}\left(-g_{\mu \nu}+\frac{D_{\mu} D_{\nu}}{M^{2}}\right)+\frac{1}{2}\left(W_{\lambda}\right)_{\mu \nu} s_{D}^{\lambda}- \\
& {\left[\frac{1}{2}\left(\left(W_{\lambda_{1}}\right)_{\mu \rho}\left(W_{\lambda_{2}}\right)_{\nu}^{\rho}+\left(W_{\lambda_{2}}\right)_{\mu \rho}\left(W_{\lambda_{1}}\right)_{\nu}^{\rho}\right)+\frac{2}{3}\left(-g_{\lambda_{1} \lambda_{2}}+\frac{D_{\lambda_{1}} D_{\lambda_{2}}}{M^{2}}\right)\left(-g_{\mu \nu}+\frac{D_{\mu} D_{\nu}}{M^{2}}\right)\right] p_{D}^{\lambda_{1} \lambda_{2}}}
\end{aligned}
$$

with $\left(W_{\lambda}\right)_{\mu \nu}=i \varepsilon_{\mu \nu \gamma \lambda} D^{\gamma} / M ; s_{D}$ the spin vector and $p_{D}$ the alignment tensor of the deuteron.

The full symmetric tensor $\Phi_{\mu \nu}(D, q)$ in Eq.(3) reads as

$$
\Phi_{\mu \nu}(D, q)=\frac{1}{4} \operatorname{Tr}\left[\bar{\Psi}_{\mu}\left(\frac{\widehat{n}+m}{m}\right)^{2} \Psi_{\nu} \frac{\widehat{p^{\prime}}-m}{m}\right]=-f_{0}\left(n^{2}\right) g_{\mu \nu}+f_{1}\left(n^{2}\right) \frac{q_{\mu} q_{\nu}}{m^{2}} .
$$

Proving Eq.(5), we introduce the modified vertex, $\Psi_{\mu}(D, q)$ :

$$
\Psi_{\mu}(D, q)=\frac{\Gamma_{\mu}(D, q)}{m^{2}-n^{2}-i 0}=\varphi_{1}\left(n^{2}\right) \gamma_{\mu}+\varphi_{2}\left(n^{2}\right) \frac{n_{\mu}}{m}+\frac{\widehat{n}-m}{m}\left(\varphi_{3}\left(n^{2}\right) \gamma_{\mu}+\varphi_{4}\left(n^{2}\right) \frac{n_{\mu}}{m}\right) .
$$

Substituting Eq.(6) into Eq.(5) and calculating the trace, one can find explicit forms of the invariant functions $f_{0,1}$

$$
\begin{gathered}
f_{0}\left(n^{2}\right)=\frac{M^{2}}{m^{2}}\left(\varphi_{1}-\frac{m^{2}-n^{2}}{m^{2}} \varphi_{3}\right) \varphi_{1}-\left(\frac{m^{2}-n^{2}}{m^{2}}\right)^{2}\left(\varphi_{1}-\varphi_{3}\right) \varphi_{3} \\
f_{1}\left(n^{2}\right)=-4\left\{\varphi_{1}+\varphi_{2}-\frac{m^{2}-n^{2}}{m^{2}}\left(\frac{\varphi_{2}}{2}+\varphi_{3}+\varphi_{4}\right)\right\}\left(\varphi_{1}+\varphi_{2}\right) \\
+\frac{M^{2}}{m^{2}}\left(\varphi_{2}-\frac{m^{2}-n^{2}}{m^{2}} \varphi_{4}\right) \varphi_{2}-\left(\frac{m^{2}-n^{2}}{m^{2}}\right)^{2}\left(\varphi_{2}+2 \varphi_{3}+\varphi_{4}\right) \varphi_{4} .
\end{gathered}
$$

The corresponding invariant scalar functions $\varphi_{i}\left(n^{2}\right)$ of the deuteron vertex with one on-shell nucleon can be computed in any reference frame. Let us note that in our case, when one particle is an onmass shell, only four partial amplitudes contribute to the process, namely in the $\rho$ spin classification, $u={ }^{3} \mathcal{S}_{1}^{++}, w={ }^{3} \mathcal{D}_{1}^{++}, v_{s}={ }^{1} \mathcal{P}_{1}^{-+}$and $v_{t}={ }^{3} \mathcal{P}_{1}^{-+}$. We can write $\varphi_{i}$ in the deuteron rest frame in 
order to relate them to the non-relativistic $S$-, $D$ - and $\mathcal{P}$-waves of the deuteron. In this case, the invariant functions take the following forms:

$$
\begin{aligned}
& \mathcal{N} \varphi_{1}=u-\frac{w}{\sqrt{2}}-\sqrt{\frac{3}{2}} \frac{m}{|\boldsymbol{q}|} v_{t} ; \\
& \mathcal{N} \varphi_{2}=-\frac{m}{\left(E_{\boldsymbol{q}}+m\right)} u-\frac{m\left(2 E_{\boldsymbol{q}}+m\right)}{|\boldsymbol{q}|^{2}} \frac{w}{\sqrt{2}}+\sqrt{\frac{3}{2}} \frac{m}{|\boldsymbol{q}|} v_{t} ; \\
& \mathcal{N} \varphi_{3}=-\sqrt{\frac{3}{2} \frac{m E_{\boldsymbol{q}}}{|\boldsymbol{q}|\left(2 E_{\boldsymbol{q}}-M\right)} v_{t} ;} \\
& \mathcal{N} \varphi_{4}=\frac{m^{2}}{M\left(E_{\boldsymbol{q}}+m\right)} u-\frac{m^{2}\left(E_{\boldsymbol{q}}+2 m\right)}{M|\boldsymbol{q}|^{2}} \frac{w}{\sqrt{2}}-\sqrt{3} \frac{m^{2}}{|\boldsymbol{q}|\left(2 E_{\boldsymbol{q}}-M\right)} v_{s},
\end{aligned}
$$

where all the vertex functions are determined in the deuteron rest frame and all the kinematic variables in Eqs.(8) have to be evaluated in this system; $E_{\boldsymbol{q}}=\sqrt{|\boldsymbol{q}|^{2}+m^{2}}$. The normalization factor $\mathcal{N}^{-1}=\pi \sqrt{2 / M}$ is chosen according to non-relativistic normalization $D W F$ : 円

$$
\int_{0}^{\infty}|\boldsymbol{q}|^{2} d|\boldsymbol{q}|\left(u^{2}(|\boldsymbol{q}|)+w^{2}(|\boldsymbol{q}|)\right)=1
$$

The relativistic invariant functions $f_{0,1}(|\boldsymbol{q}|)$ (7) can be rewritten in terms of these spin-orbit momentum wave functions as

$$
\begin{gathered}
f_{0}(|\boldsymbol{q}|)=\mathcal{N}^{-2} \frac{M^{2}}{m^{2}}\left\{\left(u-\frac{w}{\sqrt{2}}\right)^{2}+\sqrt{6} \frac{|\boldsymbol{q}|}{m}\left(u-\frac{w}{\sqrt{2}}\right) v_{t}-\frac{3}{2} v_{t}^{2}\right\} ; \\
\frac{2}{3} \frac{|\boldsymbol{q}|^{2}}{m^{2}} f_{1}(|\boldsymbol{q}|)=\mathcal{N}^{-2} \frac{M^{2}}{m^{2}}\left\{2 \sqrt{2} u w+w^{2}+v_{t}^{2}-2 v_{s}^{2}-\frac{4}{\sqrt{3}} \frac{|\boldsymbol{q}|}{m}\left[\left(u-\frac{w}{\sqrt{2}}\right) \frac{v_{t}}{\sqrt{2}}+(u+\sqrt{2} w) v_{s}\right]\right\} .
\end{gathered}
$$

Then, all the observables can be computed in terms of positive- and negative-energy wave functions $u, w$ and $v_{s}, v_{t}$, respectively. The contribution of the positive-energy waves $u, w$ to the observables results in the non-relativistic limit. The parts containing the negative-energy waves $v_{s}, v_{t}$ have a pure relativistic origin, and consequently they manifest genuine relativistic correction effects.

Using an explicit form of the density matrix (4), one can write

$$
\Phi \equiv \rho_{\mu \nu} \Phi^{\mu \nu}=\Phi^{(u)}+\Phi_{\lambda}^{(v)} s_{D}^{\lambda}+\Phi_{\lambda_{1} \lambda_{2}}^{(t)} p_{D}^{\lambda_{1} \lambda_{2}}
$$

The superscripts $(u, v, t)$ denote unpolarized, vector polarized and tensor polarized cases, respectively:

$$
\begin{aligned}
& \Phi^{(u)}(\boldsymbol{q})=f_{0}+\frac{1}{3} \frac{|\boldsymbol{q}|^{2}}{m^{2}} f_{1} \\
& \Phi_{\lambda}^{(v)}(\boldsymbol{q})=0 ; \\
& \Phi_{\lambda_{1} \lambda_{2}}^{(t)}(\boldsymbol{q})=\left[\frac{1}{3} \frac{|\boldsymbol{q}|^{2}}{m^{2}}\left(-g_{\lambda_{1} \lambda_{2}}+\frac{D_{\lambda_{1}} D_{\lambda_{2}}}{M^{2}}\right)-\left(-g_{\lambda_{1} \mu}+\frac{D_{\lambda_{1}} D_{\mu}}{M^{2}}\right)\left(-g_{\lambda_{2} \nu}+\frac{D_{\lambda_{2}} D_{\nu}}{M^{2}}\right) \frac{q^{\mu} q^{\nu}}{m^{2}}\right] f_{1} .
\end{aligned}
$$

${ }^{1}$ Note, the Gross definition $\left[16\right.$ of $\mathrm{DV} \widetilde{\Psi}_{\mu}(q)$ is related to the BS vertex $\Psi_{\mu}(q)$ (6) as the follows:

$$
\widetilde{\Psi}_{\mu}(q)=\Psi_{\mu}(-q) .
$$

Comparing it with Eqs.(46) of Ref. [16], one can see that the Gross wave functions $\widetilde{u}(q), \widetilde{w}(q)$ and $\widetilde{v}_{s}(q), \widetilde{v}_{t}(q)$ are connected with our wave functions (8) as

$$
\begin{aligned}
& \widetilde{u}\left(q_{0},|\boldsymbol{q}|\right)=u\left(-q_{0},|\boldsymbol{q}|\right) ; \quad \widetilde{w}\left(q_{0},|\boldsymbol{q}|\right)=w\left(-q_{0},|\boldsymbol{q}|\right) ; \\
& \widetilde{v}_{s}\left(q_{0},|\boldsymbol{q}|\right)=-v_{s}\left(-q_{0},|\boldsymbol{q}|\right) ; \quad \widetilde{v}_{t}\left(p_{0},|\boldsymbol{p}|\right)=-v_{t}\left(-q_{0},|\boldsymbol{p}|\right),
\end{aligned}
$$

where $q_{0}=M / 2-E_{\boldsymbol{q}}$. 
Let us consider now the case when the deuteron has a tensor polarization. If the initial deuteron is only aligned due to the $p_{D}^{z z}$ component, then the inclusive spectrum of the reaction $\boldsymbol{D}+p \rightarrow \pi+X$ (3) can be written in the form:

$$
\rho_{p D}^{\pi}\left(p_{D}^{z z}\right)=\rho_{p D}^{\pi}\left[1+\mathcal{A}_{z z} \cdot p_{D}^{z z}\right]
$$

where $\rho_{p D}^{\pi}$ is the inclusive spectrum for the case of unpolarized deuterons and $\mathcal{A}_{z z} \equiv \sqrt{2} T_{20}(-\sqrt{2} \leq$ $T_{20} \geq 1 / \sqrt{2}$ ) is the tensor analyzing power. One can write:

$$
\begin{aligned}
\rho_{p D}^{\pi} & =\frac{1}{(2 \pi)^{3}} \int \frac{\sqrt{\lambda(p, n)}}{\sqrt{\lambda(p, D)}}\left[\rho_{p N}^{\pi} \cdot \Phi^{(u)}(|\boldsymbol{q}|)\right] \frac{m^{2} d^{3} q}{E_{\boldsymbol{q}}} \\
\rho_{p D}^{\pi} \cdot \mathcal{A}_{z z} & =-\frac{1}{(2 \pi)^{3}} \int \frac{\sqrt{\lambda(p, n)}}{\sqrt{\lambda(p, D)}}\left[\rho_{p N}^{\pi} \cdot \Phi^{(t)}(|\boldsymbol{q}|)\right]\left(\frac{3 \operatorname{Cos}^{2} \vartheta_{\boldsymbol{q}}-1}{2}\right) \frac{m^{2} d^{3} q}{E_{\boldsymbol{q}}} .
\end{aligned}
$$

where

$$
\begin{aligned}
\Phi^{(u)}(|\boldsymbol{q}|) & =f_{0}(|\boldsymbol{q}|)+\frac{1}{3} \frac{|\boldsymbol{q}|^{2}}{m^{2}} f_{1}(|\boldsymbol{q}|) \\
& =\mathcal{N}^{-2} \frac{M^{2}}{m^{2}}\left\{u^{2}+w^{2}-v_{t}^{2}-v_{s}^{2}+\frac{2}{\sqrt{3}} \frac{|\boldsymbol{q}|}{m}\left[\left(\sqrt{2} v_{t}-v_{s}\right) u-\left(v_{t}+\sqrt{2} v_{s}\right) w\right]\right\} \\
\Phi^{(t)}(|\boldsymbol{q}|) & =\frac{2}{3} \frac{|\boldsymbol{q}|^{2}}{m^{2}} f_{1}(|\boldsymbol{q}|) \\
& =\mathcal{N}^{-2} \frac{M^{2}}{m^{2}}\left\{2 \sqrt{2} u w+w^{2}+v_{t}^{2}-2 v_{s}^{2}-\frac{4}{\sqrt{3}} \frac{|\boldsymbol{q}|}{m}\left[\left(u-\frac{w}{\sqrt{2}}\right) \frac{v_{t}}{\sqrt{2}}+(u+\sqrt{2} w) v_{s}\right]\right\}
\end{aligned}
$$

It is intuitively clear that the nucleons in the deuteron are mainly in the states with angular momenta $L=0,2$ (see also a numerical analysis of the solutions of the $B S$ equation in terms of amplitudes within the $\rho$-spin basis [17]), the probability of states with $L=1\left(v_{s, t}\right)$ in Eqs.(17,,18) is much smaller than the probability for the $u, w$ configurations. Moreover, it can be shown that the $u$ and $w$ waves directly correspond to the non-relativistic $\mathcal{S}$ and $\mathcal{D}$ ones. Therefore, Eqs.(17, 18) with only $u, w$ waves can be identified as main contributions to the corresponding observables, and they can be compared with their non-relativistic analogies. The other terms possessing contributions from $\mathcal{P}$-waves are proportional to $\boldsymbol{q} / m$ (the diagonal terms in $v_{s, t}$ are negligible). Due to their pure relativistic origin, one can refer to them as relativistic corrections.

Let us consider a minimal relativization scheme which describes rather well the differential cross section for such a process as deuteron break-up $A(D, p) X$. The minimal relativization procedure [18, 2] comprises the following (i) replacing the argument of the non-relativistic wave functions by the light-cone variable $\boldsymbol{k}=\left(\boldsymbol{k}_{\perp}, \boldsymbol{k}_{\|}\right)$

$$
\boldsymbol{k}^{2}=\frac{m^{2}+\boldsymbol{k}_{\perp}^{2}}{4 x(1-x)}-m^{2} ; \quad k_{\|}=\sqrt{\frac{m^{2}+\boldsymbol{k}_{\perp}^{2}}{x(1-x)}}\left(\frac{1}{2}-x\right) .
$$

where $x=\left(E_{\boldsymbol{q}}+|\boldsymbol{q}| \operatorname{Cos} \vartheta_{\boldsymbol{q}}\right) / M=\left(\varepsilon^{\prime}-p_{\|}^{\prime}\right) / M ;\left|\boldsymbol{k}_{\perp}\right|=p_{\perp}^{\prime}$ in the deuteron rest frame, and (ii) multiplying the wave functions by the factor $\sim 1 /(1-x)$. It results in a shift of the argument towards larger values, and the wave function itself decreases more rapidly. This effect of suppressing the wave function is compensated by the kinematic factor $1 /(1-x)$.

In the $B S$ approach the relativistic effects are of a dynamic nature [19] and not reduced to a simple shift in arguments. In addition to $\mathcal{S}$ and $\mathcal{D}$ waves, it contains negative energy components, i.e., $\mathcal{P}$ waves. One can see that they play a more important role in the polarization case and lead to an improvement of description of the data. 


\section{RESULTS AND DISCUSSION}

Below, the calculated results of the inclusive relativistic invariant pion spectrum and the tensor analyzing power in the fragmentation process $D p \rightarrow \pi X$ are presented and compared with the available experimental data [1, 10]. These experimental data are presented as a functions of the so-called cumulative scaling variable $x_{\mathcal{C}}$ ("cumulative number") [20]. For our reaction, this variable is defined as follows:

$$
x_{\mathcal{C}}=2 \frac{(p \pi)-\mu^{2} / 2}{(D p)-M m-(D \pi)}=2 \frac{t-m^{2}}{\left(t-m^{2}\right)+(M+m)^{2}-s_{X}} \leq 2 .
$$

In the rest frame of the deuteron $D=(M, \mathbf{0})$, it can be rewritten in the form:

$$
x_{\mathcal{C}}=2 \frac{E E_{\pi}-p p_{\pi} \operatorname{Cos}_{\pi}-\mu^{2} / 2}{M\left(E-E_{\pi}-m\right)} \rightarrow 2 \frac{E}{T_{p}} \frac{\alpha}{1-E_{\pi} / T_{p}},
$$

where $\alpha=\left(E_{\pi}-\pi \operatorname{Cos} \vartheta_{\pi}\right) / M$ is a light-cone variable. The value of $x_{\mathcal{C}}$ corresponds to a minimum mass (in nucleon mass units) of part of the projectile nucleus (deuteron) involved in the reaction. The value of $x_{\mathcal{C}}$ larger than 1 corresponds to the a cumulative pion.

Deuteron (polarized and unpolarized) fragmentation into proton $D+A \rightarrow p\left(0^{\circ}\right)+X$ is one of the more intensively studied reaction with hadronic probe. The reason of this study is, first, a big cross section and, second, rather a simple relation of the inclusive spectrum and polarization observables to the $\mathcal{S}$ - and $\mathcal{D}$-waves of the DWF obtained within IA. For example, the tensor analyzing power $T_{20}$ within IA can be written in the following simple form:

$$
T_{20}=-\frac{1}{\sqrt{2}} \frac{2 \sqrt{2} u w+w^{2}}{u^{2}+w^{2}}
$$

This relation does not depend on the amplitude of elementary reaction $p n \rightarrow p X$ which is taken in IA as an off-mass shell. As shown in [7], both the differential cross section and $T_{20}$ for fragmentation $D+p \rightarrow p\left(0^{\circ}\right)+X$ can be described within the IA at $k \leq 0.2 \mathrm{GeV} / \mathrm{c}$ only. At larger momenta $k$, secondary interactions, in particular the triangle graphs with a virtual pion, have to be taken into account in order to describe these observables least satisfactorily.

However, as shown in [21] for the pion production $D+p \rightarrow \pi\left(0^{\circ}\right)+X$ at the cumulative over region the rescattering mechanism is kinematically suppressed. Therefore, one can use the IA only by the theoretical calculus of the differential cross section and tensor analyzing power $T_{20}$ for this reaction. The calculated results of the invariant spectrum of pions produced via by the $D+p \rightarrow \pi\left(0^{\circ}\right)+X$ reaction are presented in Fig. (1-2). The vertex $N N \rightarrow \pi Y$ is taken as an on-mass shell and corresponding differential cross section proposed in [22] was used.

A large sensitivity of the inclusive spectrum to this vertex and a small one to the type of the nonrelativistic DWF can be seen from Fig. 1. The Fig. 2 shows that the inclusion of the $\mathcal{P}$-waves contribution to the DWF within the Bethe-Salpeter or Gross approaches results in a better (but not satisfactory) description of the experimental data over the cumulative region. From Fig. 3 one can see the effects of calculation using the minimal relativization scheme [2].

The calculated results of $T_{20}$ for the reaction of polarized deuteron fragmentation into cumulative pions are shown in Fig. (4-6). From these figures one can see a small sensitivity of $T_{20}$ to the vertex corresponding to the $N N \rightarrow \pi Y$ process. It is also seen that $T_{20}$ is more sensitive to the DWF form than the invariant spectrum. The experimental data on $T_{20}$ are not described by any DWF used in this paper. Note that there may be an alternative approach to study the deuteron structure at small distances which assumes a possible existence of non-nucleon or quark degrees of freedom [7, 23, 24] in the deuteron and nucleus. For example, according to [2], large momenta of nucleons can due to few nucleon correlations in the nucleus. Then the deuteron structure can be described by assuming the quark degrees of freedom [13, 14]. On the other hand, the shape of a high momentum tail of the 
nucleon distribution in the deuteron can be constructed from the basis of its true Regge asymptotic [12], and the corresponding parameters can be found from a good description of the inclusive proton spectrum in deuteron fragmentation $D p \rightarrow p X$ [12, 7]. According to [12, 7], one can write the following form for $\widetilde{\Phi}^{(u)}(|\boldsymbol{q}|)$, see Eq.(17):

$$
\widetilde{\Phi}^{(u)}(|\boldsymbol{q}|)=\mathcal{N}^{-1} \frac{M^{2}}{m^{2}}\left\{\left(1-\alpha_{6 q}\right) \cdot\left[u^{2}(|\boldsymbol{q}|)+w^{2}(|\boldsymbol{q}|)\right]+\alpha_{6 q} \frac{4 \pi x}{E_{\boldsymbol{q}}} \cdot G_{6 q}\left(x, \boldsymbol{k}_{\perp}\right)\right\}
$$

where the parameter $\alpha_{6 q}$ is the probability for a non-nucleon component in the deuteron which is a state of two colorless $(3 q)$ systems.

$$
G_{6 q}\left(x, \boldsymbol{k}_{\perp}\right)=\frac{b^{2}}{2 \pi} \cdot \frac{\Gamma(A+B+2)}{\Gamma(A+1) \Gamma(B+1)} \cdot x^{A}(1-x)^{B} \cdot e^{-b k_{\perp}} .
$$

Fig. 7. presents the invariant pion spectrum calculated within the relativistic impulse approximation including the non-nucleon component in the DWF [12, 7]; its probability $\alpha_{6 q}$ is $0.2-0.4$ (dot-dashed and dashed curves, respectively). One can see a good description of the experimental data [1] at all $x_{\mathcal{C}}$.

\section{SUMMARY AND OUTLOOK}

The main goal of this paper is to study the reaction of deuteron fragmentation into pions within the framework of the nucleon model of deuteron. Main results can be summarized as follows.

1. It is quite incorrect to use the nonrelativistic deuteron wave function for the analysis of $D-N$ fragmentation into hadrons, in particular pions. Relativistic effects are sizable, especially in the kinematic region corresponding to short intra-deuteron distances or large $x$. It is seen from the behavior of the inclusive pion spectrum and particularly the tensor analyzing power $T_{20}$ at large $x$.

2. At the present time, the state of theory is such, that the unique procedure to include relativistic effects in the deuteron has not been found yet. An extreme sensitivity to different methods of the relativization deuteron wave function is found for $T_{20}$ at $x \geq 1$.

3. A large sensitivity of the inclusive spectrum of pions to the vertex of the $N N \rightarrow \pi X$ process can be seen from Fig. 1. In contrast to this the small sensitivity of $T_{20}$ to this vertex is found, as seen from Fig. 4. This polarization observable is very sensitive to the DWF form (Fig. (4-6)).

4. Very interesting experimental data on $T_{20}$ showing approximately zero values at $x_{\mathcal{C}} \geq 1$ are not reproduced by a theoretical calculus using even different kinds of the relativistic DWF. This may indicate a possible existence of non-nucleon degrees of freedom or basically new mechanism of pion production in the kinematic region forbidden for free $N-N$ scattering.

5. For the deuterons fragmentation into protons emitted forward, the tensor analyzing power $T_{20}$ is not described by the standard nuclear physics using the nucleon degrees of freedom at $x_{\mathcal{C}} \geq 1.7$ [7]. On the contrary, $T_{20}$ for the fragmentation $D p \rightarrow \pi X$ can not be described within the same assumptions over all region $x_{\mathcal{C}} \geq 1$. Note that experimental and theoretical study of polarization observables of deuteron fragmentation into hadrons containing of different quarks is powerful tool for understanding the deuteron structure at small distances. 


\section{References}

[1] A.M. Baldin, Nucl.Phys. A434 (1985) 695c.

[2] L.L. Frankfurt and M.I. Strikman, Phys. Rep. 76 (1981) 215.

[3] P. Bosted et al., Phys. Rev. Lett. 49 (1982) 1380.

[4] Vina Punjabi and Charles F. Perdrisat, in Proc. of the 3rd International Symposium "DUBNA DEUTERON-95", Dubna, 1996, 304.

[5] M. Lancome et al., Phys. Lett. B101 (1981) 139.

[6] R.V. Reid, Ann. Phys. 50 (1968) 411.

[7] G.I. Lykasov, EPAN 24 (1993) 59.

[8] V.G. Ableev et al., Nucl. Phys. A393 (1983) 491; A411 (1983) 541E; JETP Lett. 37 (1983), 233; 47 (1988), 279.

[9] V.G. Ableev et al., in Proc. of the 7-th Intern. Conf. on Polarization Phenomena in Nuclear Physics (Paris 1990), Vol.1, p.40F.

[10] S.V. Afanasiev et al., Nucl. Phys. A625 (1997) 817; Phys. Lett. B445 (1998) 14; Preprint JINR E2-98-319, Dubna, 1998, 10p.

[11] N.S. Amelin and G.I. Lykasov, Sov.J.Nucl.Phys. 33 (1981) 100.

[12] A.V. Efremov, A.B. Kaidalov, V.T. Kim, G.I. Lykasov and N.V. Slavin, Sov.J.Nucl.Phys. 47 (1988) 868.

[13] V.K. Lukyanov and A.I. Titov, Sov.J.Part.Nucl. 10 (1979) 321.

[14] V.V. Burov, V.K. Lukyanov and A.I. Titov, Sov.J.Part.Nucl. 15 (1984) 558.

[15] S.G. Bondarenko, V.V. Burov, M. Beyer and S.M. Dorkin, LANL Preprint nucl-th/9606035.

[16] W.W. Buck and F. Gross, Phys. Rev. D20 (1979) 2361; Phys. Lett. B63 (1976) 286.

[17] L.P. Kaptari, B. Kaempfer, S.M. Dorkin and S.S. Semikh, LANL Preprint nucl-th/9612027.

[18] S.J. Brodsky et al., Phys. Rev. D8 (1973) 4574; J.B. Kogut and D.E. Soper, Phys. Rev. D1 (1970) 2901; S. Weinberg, Phys. Rev. 150 (1966) 1313.

[19] L.P. Kaptari, A.Yu. Umnikov, F.C. Khanna and B. Kaempfer, Phys. Lett. B351 (v.4) (1995) 400.

[20] V.S. Stavinski, Sov.J.Part.Nucl. 10 (1979) 373.

[21] L.A. Kondratyuk and V.B. Kopeliovich, JETF Lett. 21 (1975) 88.

[22] V.S. Barashenkov and N.V. Slavin, EPAN 15 (v.5) (1984) 997.

[23] L.Ya. Glozman, V.G. Neudatchin and I.T. Obukhovsky, Phys. Rev. C48 (1993) 389.

[24] A.P. Kobushkin, J, Phys. G: Nucl. Phys. 19 (1993) 1993.

[25] A.Yu. Umnikov, LANL Preprint nucl-th/9605292.

[26] F. Gross, J.W. VanOrden and Karl Holinde, Phys. Rev. C45 (1992) 2094; C41 (1990) R1909. 


\section{FIGURE CAPTIONS}

FIG. 1. : The invariant spectrum of the backward pions in the deuteron fragmentation reaction calculated in the relativistic impulse approximation using various types of the nonrelativistic DWF. The calculated results are compared with the experimental data from [1] for a the projectile proton momentum of $P_{p}=9 \mathrm{GeV} / \mathrm{c}$. The thin dashed curve corresponds to the calculus by neglecting the dependence of the elementary vertex $N N \rightarrow \pi Y$ on the relativistic invariant variables.

FIG. 2. : The invariant spectrum calculated using two forms of the relativistic DWF's, [25] and [16]. The experimental data are taken from [1]. The thin solid line corresponds to the DWF [25], where the total probability of small components: $P_{v}=\int_{0}^{\infty} p^{2} d p \cdot\left[v_{t}^{2}+v_{s}^{2}\right] \simeq 0.2 \%$. The long-dashed, dashed and thick solid lines represent the calculations with the Gross DWF using the mixing parameter $\lambda=0.0,0.4$ and 1.0, respectively [26]. This corresponds probabilities $P_{v}=$ $0.03 \%, 0.44 \%$ and $1.46 \%$ to be obtained of the small component. The dot-dashed line corresponds to the nonrelativistic Paris DWF.

FIG. 3. : The invariant pion spectrum calculated using the nonrelativistic DWF's obtained by the minimal relativization scheme (MRS) [2, 18]. The solid, dashed and long-dashed lines correspond to various DWF forms: Paris, RSC and Bonn. The experimental data are taken from [1]].

FIG. 4. : The tensor analyzing power $T_{20}$ of deuterons. The calculated results are compared with the experimental data from [10] at a projectile proton momentum $P_{p}=4.46 \mathrm{GeV} / \mathrm{c}$. The thin dashed curve corresponds to the calculus by neglecting the internal structure of the elementary vertex $N N \rightarrow \pi Y$. The solid, long-dashed, dashed and dot-dashed lines correspond to the calculus using various types of the nonrelativistic DWF: Paris, RSC and two Bonn types - relativistic Bonn DWF and full Bonn DWF, respectively.

FIG. 5. : $T_{20}$ calculated using two forms of the relativistic DWF's, 25] and [16]. Notation as in Fig. 2. The experimental data are taken from [10].

FIG. 6. : $T_{20}$ calculated with the nonrelativistic DWF's using the minimal relativization scheme (MRS) 22, 18]. The solid, thick dashed, thin dashed and long-dashed lines correspond to the various DWF forms: Paris, the RSC and two Bonn DWF's - full and relativistic. The experimental data are taken from [10].

FIG. 7. : The invariant pion spectrum calculated within the relativistic impulse approximation with including of the non-nucleon component in the DWF [12, 7]; its probability $\alpha_{6 q}$ is $0.2-0.4$ (dotdashed and dashed curves, respectively). One can a the good description of the experimental data [1] for all $x_{\mathcal{C}}$. 


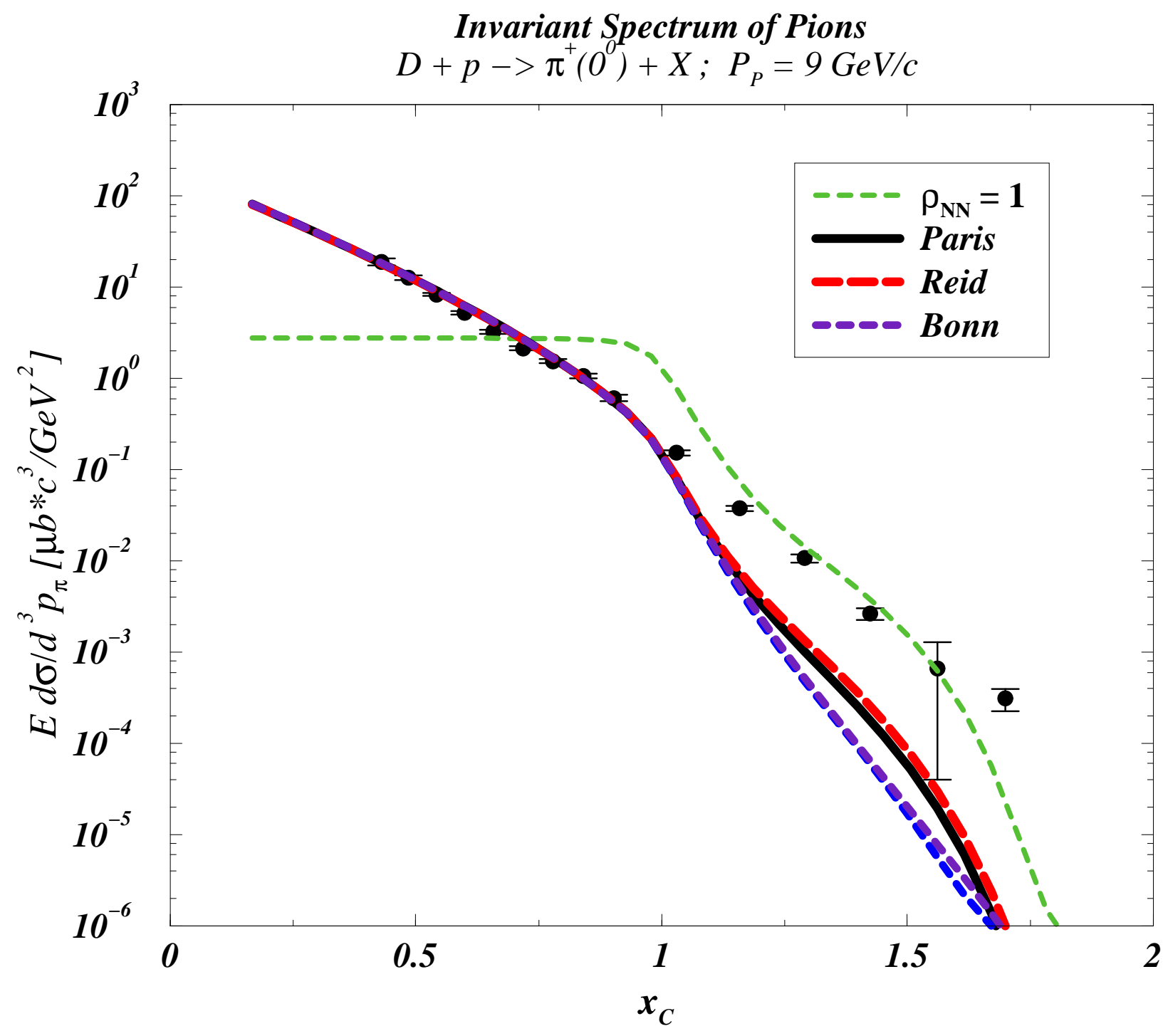

FIG. 1. 


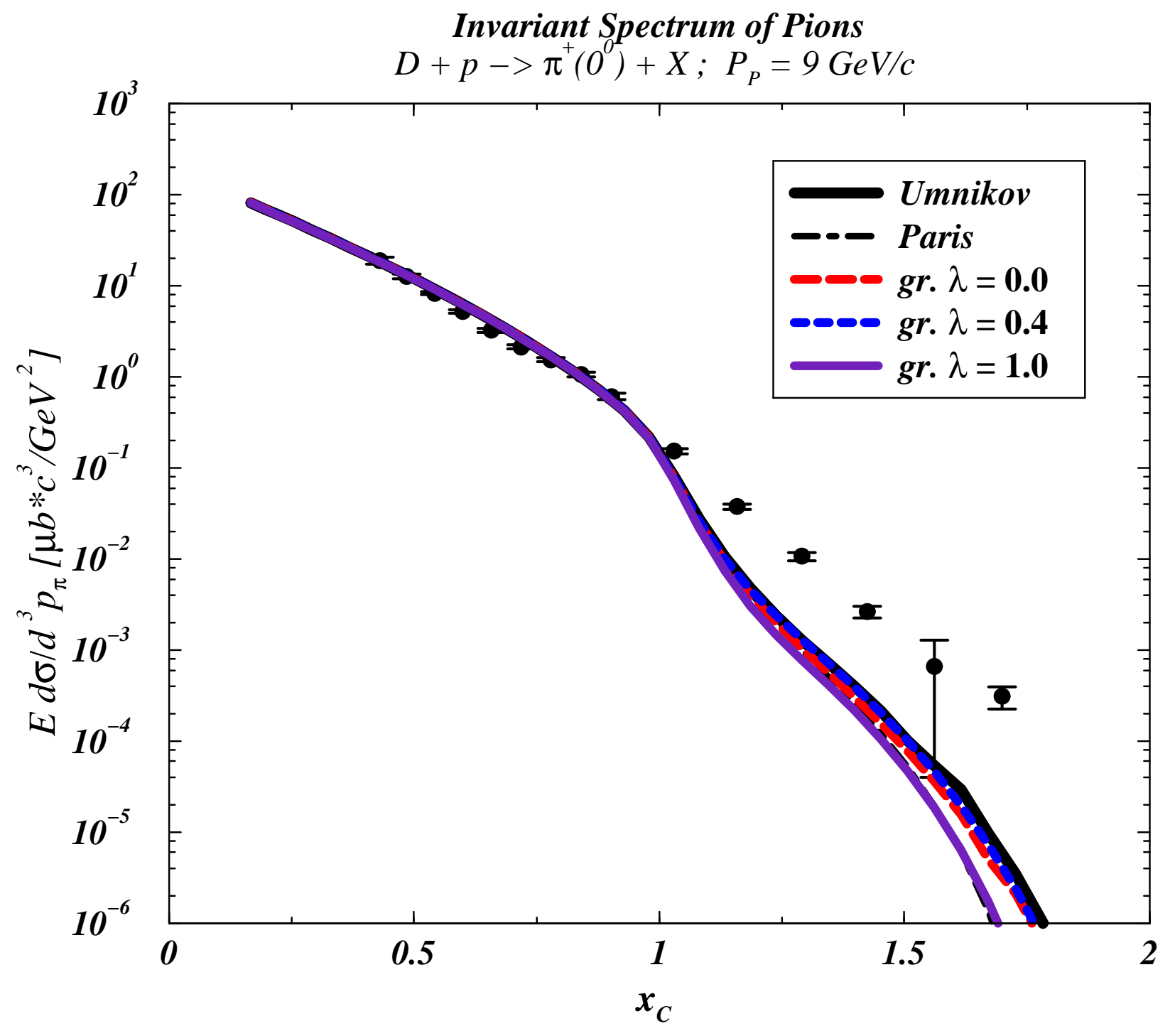

FIG. 2. 


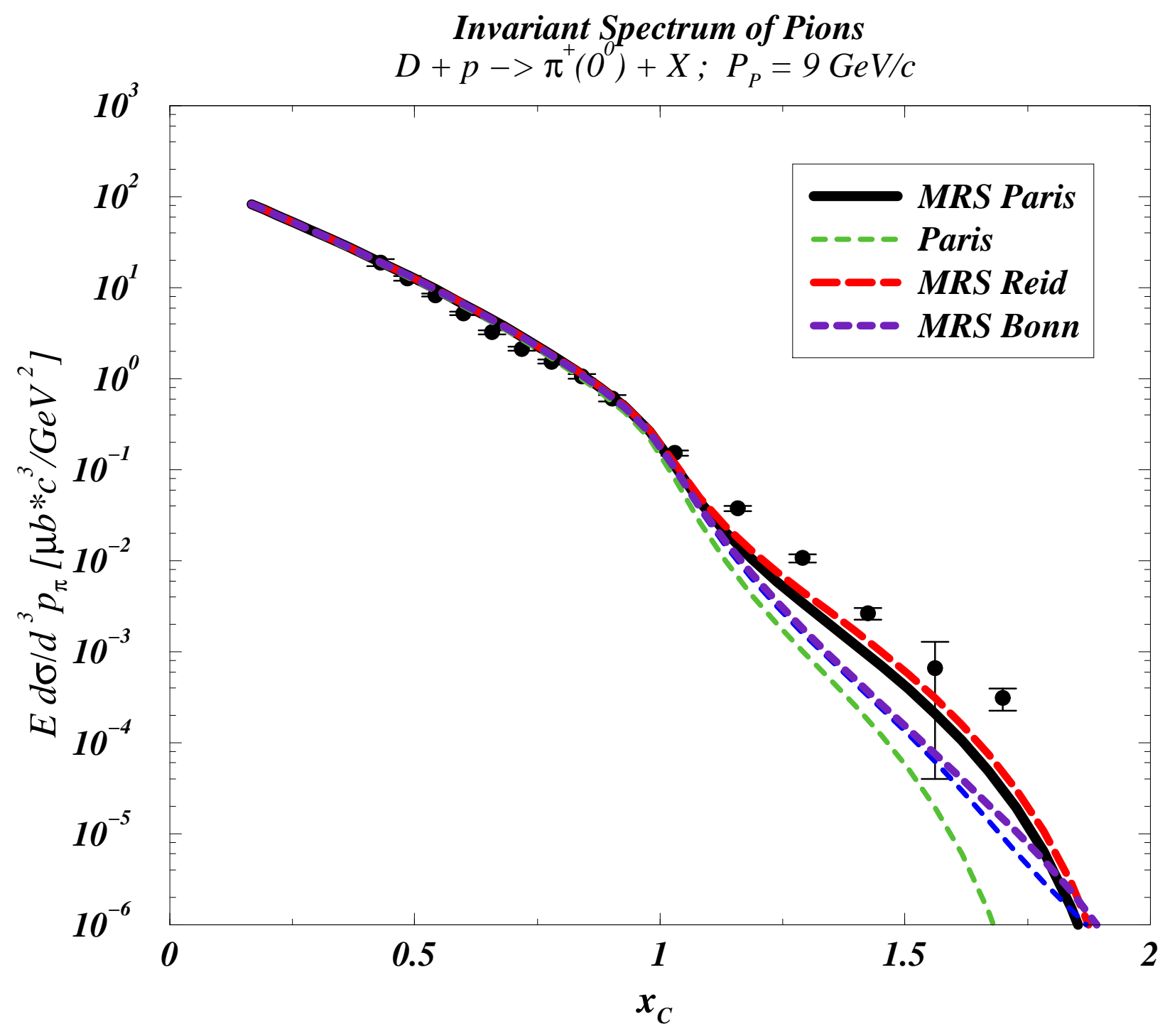

FIG. 3. 


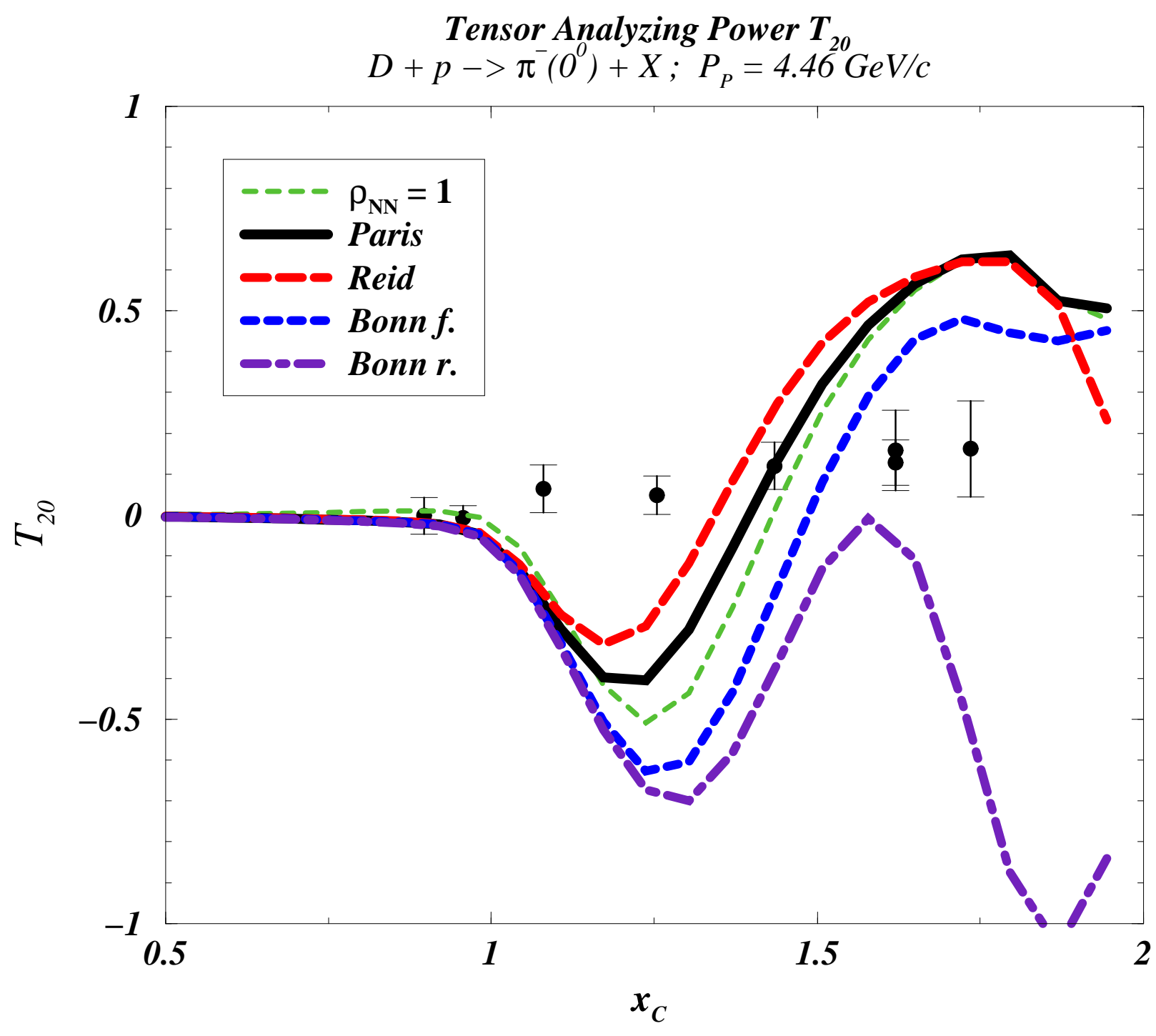

FIG. 4. 


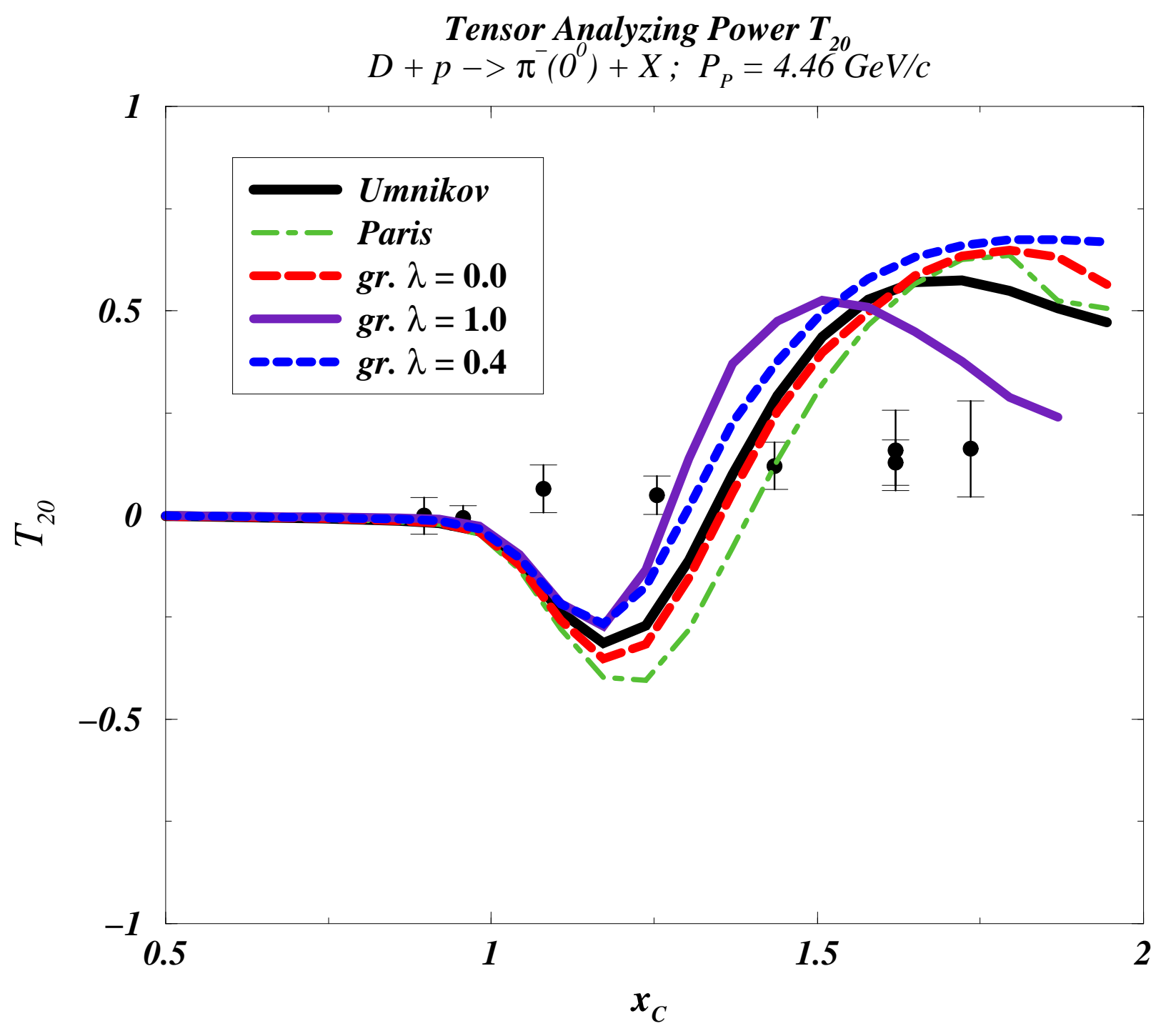

FIG. 5. 


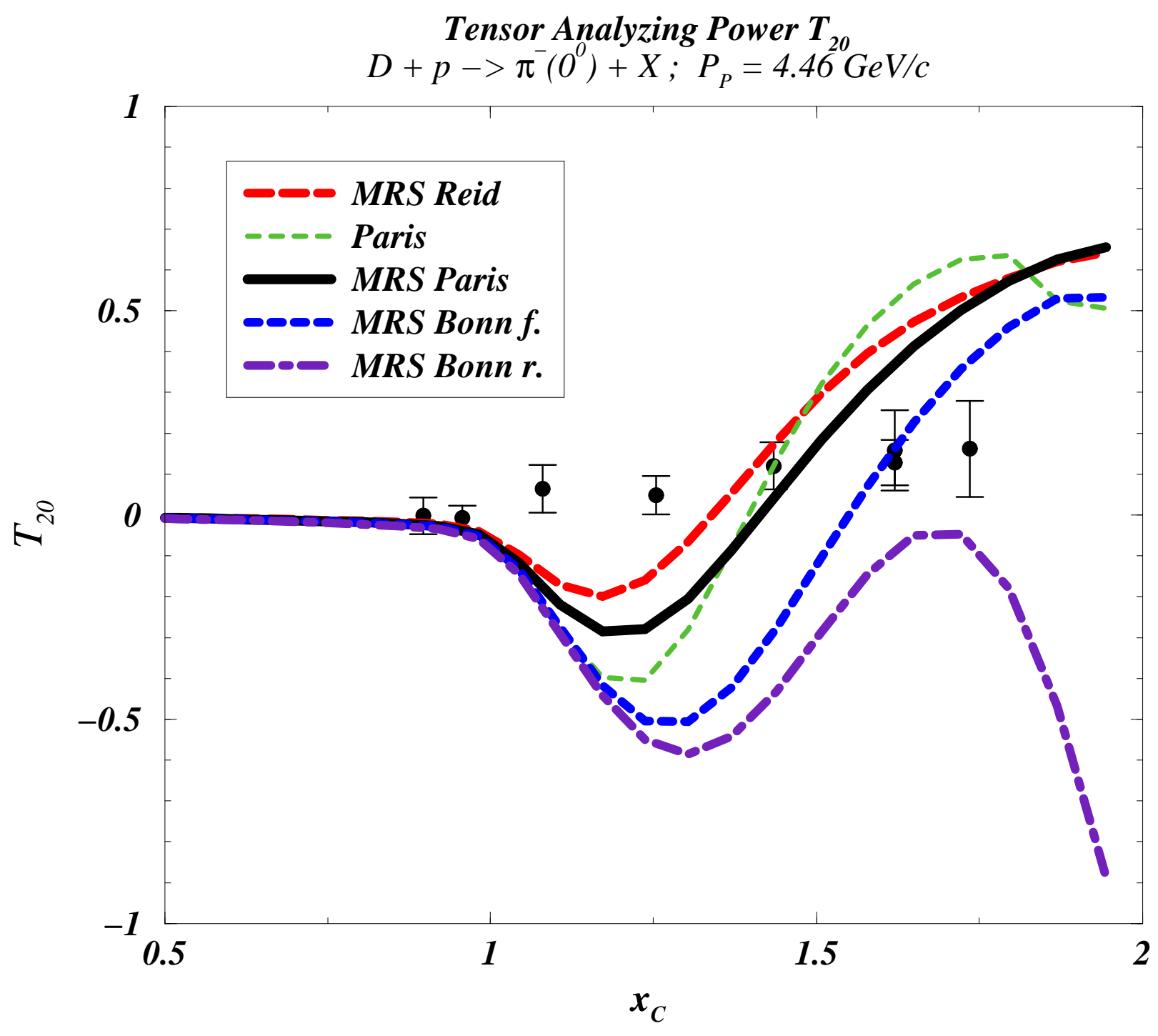

FIG. 6. 


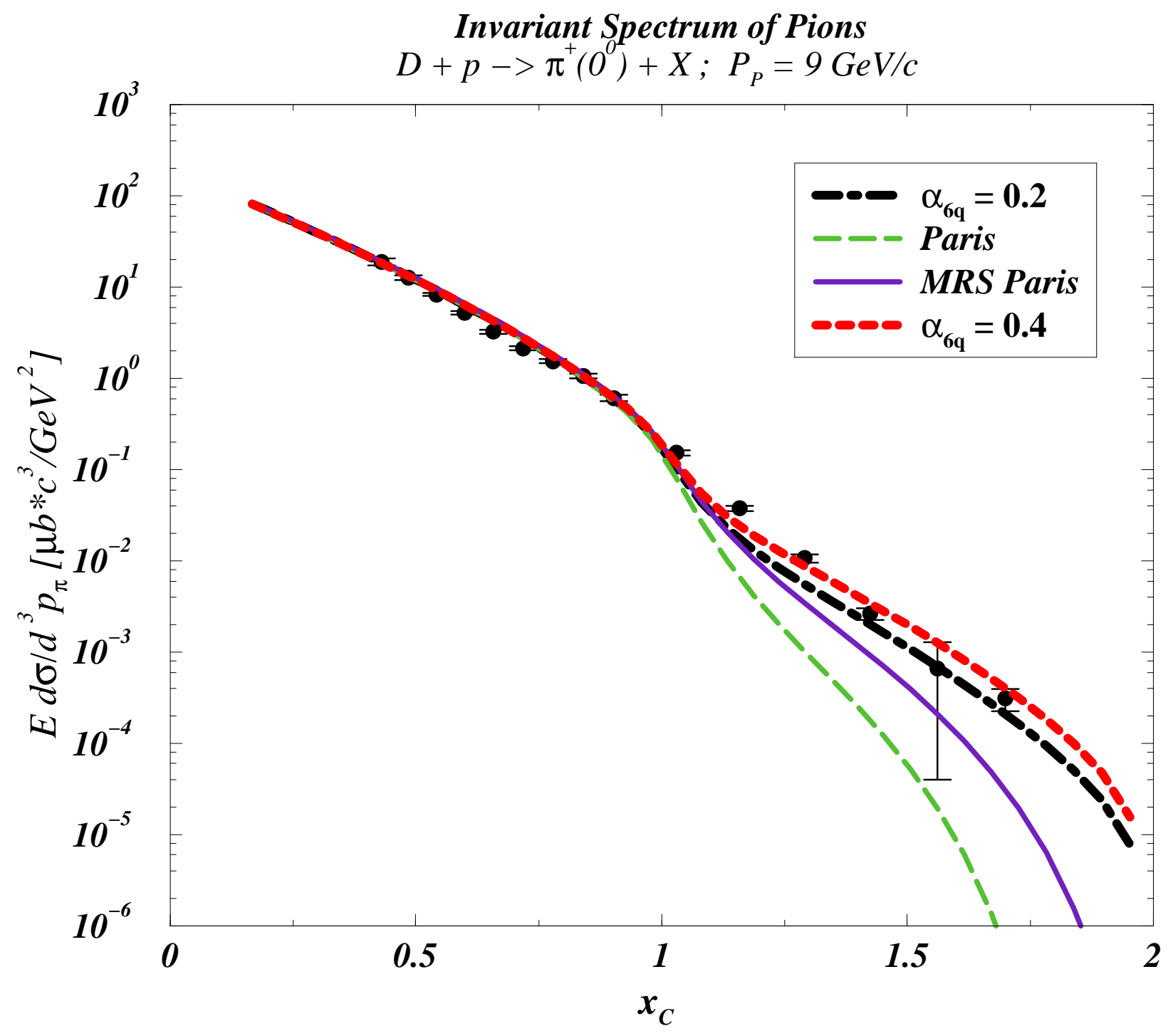

FIG. 7. 


\section{POLARIZATION PHENOMENA IN FRAGMENTATION PROCESSES OF TYPE $\boldsymbol{D}+p \rightarrow \pi+X$}

A.Yu. Illarionov and A.G. Litvinenko,

Laboratory of High Energy

G.I. Lykasov

Laboratory of Nuclear Problems

July 17-22, 2000 


\section{Outline}

- The limiting fragmentation of deuterons into pions emitted at zero angle is analyzed.

- The inclusive relativistic invariant spectrum of pions and the tensor analyzing power $T_{20}$ are investigated within framework of the impulse approximation using the different kinds of the deuteron wave function (DWF).

- The influence of the inclusion of the P-wave in the DWF is studied, too.

- It is shown that the invariant spectrum is more sensitive to the amplitude of the $N N \rightarrow \pi X$ process than the tensor analyzing power $T_{20}$.

- Our results are compared with experimental data and non-relativistic calculations and with the outcome of a relativization procedure of the DWF.

\section{Prague'2000}




\section{Impulse Approximation}

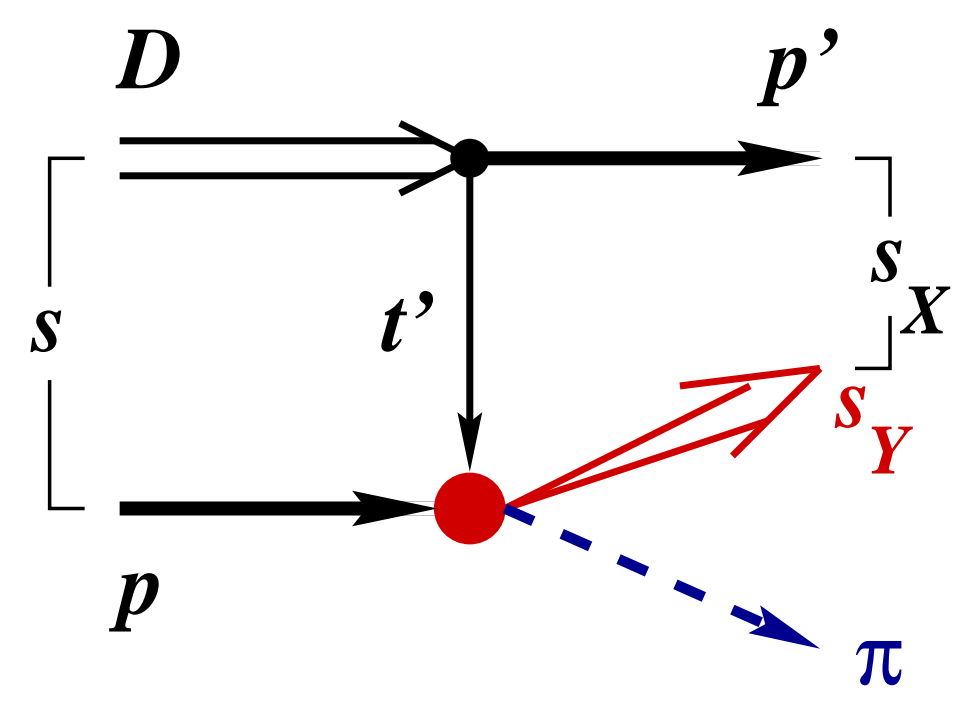

If the initial deuteron is only aligned due to $p_{D}^{z z}$ component, then the inclusive spectrum of our reaction reads

$$
\rho_{p D}^{\pi}\left(p_{D}^{z z}\right)=\rho_{p D}^{\pi}\left[1+\mathcal{A}_{z z} \cdot p_{D}^{z z}\right]
$$

where $\rho_{p D}^{\pi}$ is the inclusive spectrum for the case of unpolarized deuterons and $\mathcal{A}_{z z} \equiv \sqrt{2} T_{20}$ is the tensor analyzing power. 
They explicit forms are

$$
\begin{aligned}
& \rho_{p D}^{\pi}= \frac{1}{(2 \pi)^{3}} \int \frac{\sqrt{\lambda(p, n)}}{\sqrt{\lambda(p, D)}}\left[\rho_{p N}^{\pi} \cdot \Phi^{(u)}(|\boldsymbol{q}|)\right] \frac{d^{3} q}{E_{\boldsymbol{q}}} \\
& \rho_{p D}^{\pi} \cdot \mathcal{A}_{z z}=-\frac{1}{(2 \pi)^{3}} \int \frac{\sqrt{\lambda(p, n)}}{\sqrt{\lambda(p, D)}}\left[\rho_{p N}^{\pi} \cdot \Phi^{(t)}(|\boldsymbol{q}|)\right] \\
& \times\left(\frac{3 \operatorname{Cos}^{2} \vartheta \boldsymbol{q}-1}{2}\right) \frac{d^{3} q}{E_{\boldsymbol{q}}}
\end{aligned}
$$

where

$$
\begin{gathered}
\Phi^{(u)}(|q|)=\mathcal{N}^{-2}\left\{u^{2}+w^{2}-v_{t}^{2}-v_{s}^{2}\right. \\
\left.+\frac{2}{\sqrt{3}} \frac{|\boldsymbol{q}|}{m}\left[\left(\sqrt{2} v_{t}-v_{s}\right) u-\left(v_{t}+\sqrt{2} v_{s}\right) w\right]\right\}, \\
\Phi^{(t)}(|\boldsymbol{q}|)=\mathcal{N}^{-2}\left\{2 \sqrt{2} u w+w^{2}+v_{t}^{2}-2 v_{s}^{2}\right. \\
\left.-\frac{4}{\sqrt{3}} \frac{|\boldsymbol{q}|}{m}\left[\left(u-\frac{w}{\sqrt{2}}\right) \frac{v_{t}}{\sqrt{2}}+(u+\sqrt{2} w) v_{s}\right]\right\} .
\end{gathered}
$$




\section{Kinematics}

The cumulative scaling variable, $x_{\mathcal{C}}$ is define by

$$
x_{\mathcal{C}}=2 \frac{(p \pi)-\mu^{2} / 2}{(D p)-M m-(D \pi)} \leq 2
$$

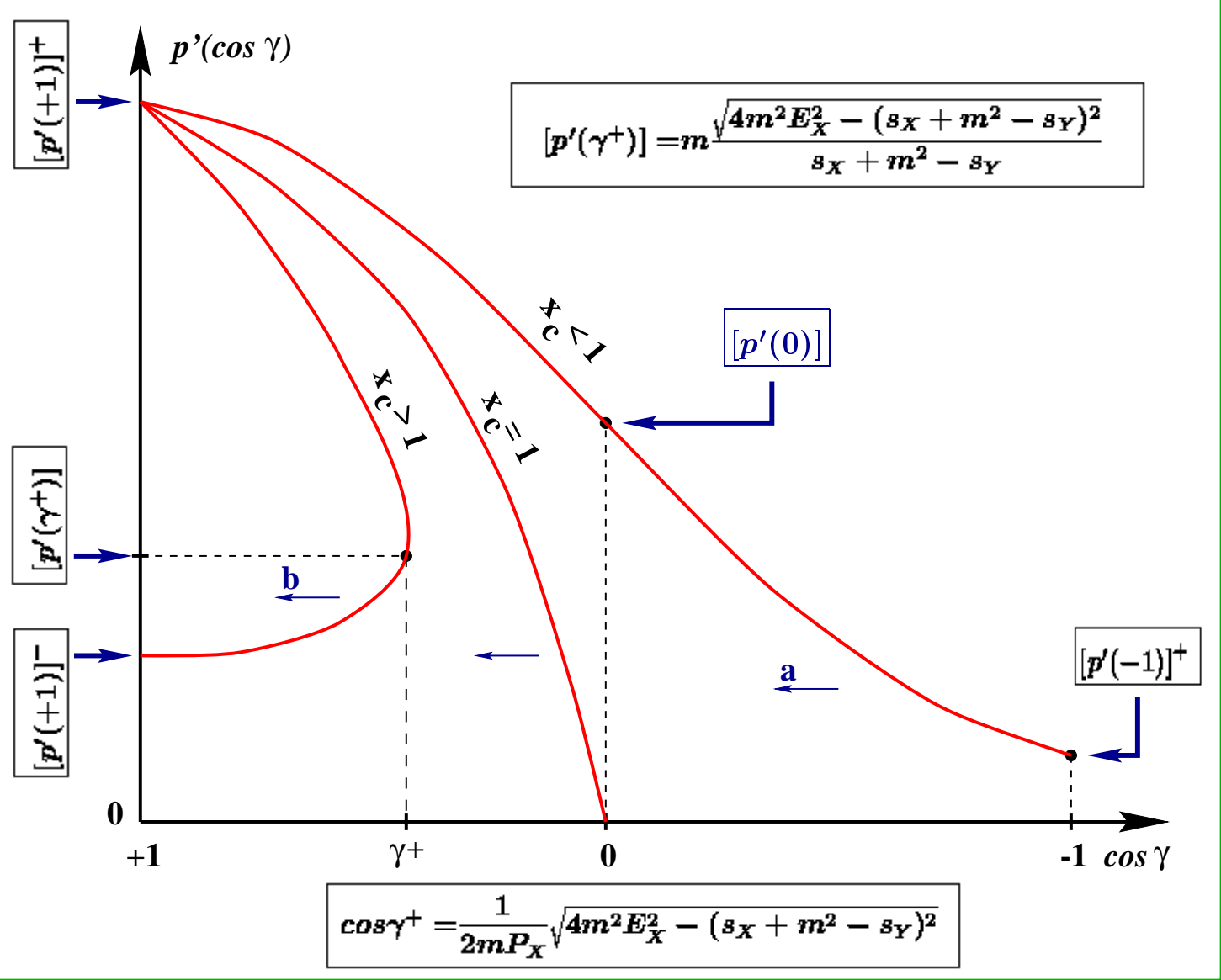

\begin{tabular}{||c||c|c|c|c|c||}
\hline \hline$x_{\mathcal{C}}$ & $0.0-1.0$ & 1.25 & 1.5 & 1.75 & 1.9 \\
\hline$k_{\min }\left(P_{p}=4.46 \mathrm{GeV}\right)$ & 0.0 & 0.16 & 0.32 & 0.54 & 0.80 \\
\hline$k_{\min }\left(P_{p}=9.00 \mathrm{GeV}\right)$ & 0.0 & 0.19 & 0.40 & 0.71 & 1.15 \\
\hline
\end{tabular}

Prague'2000

Alexei Illarionov 

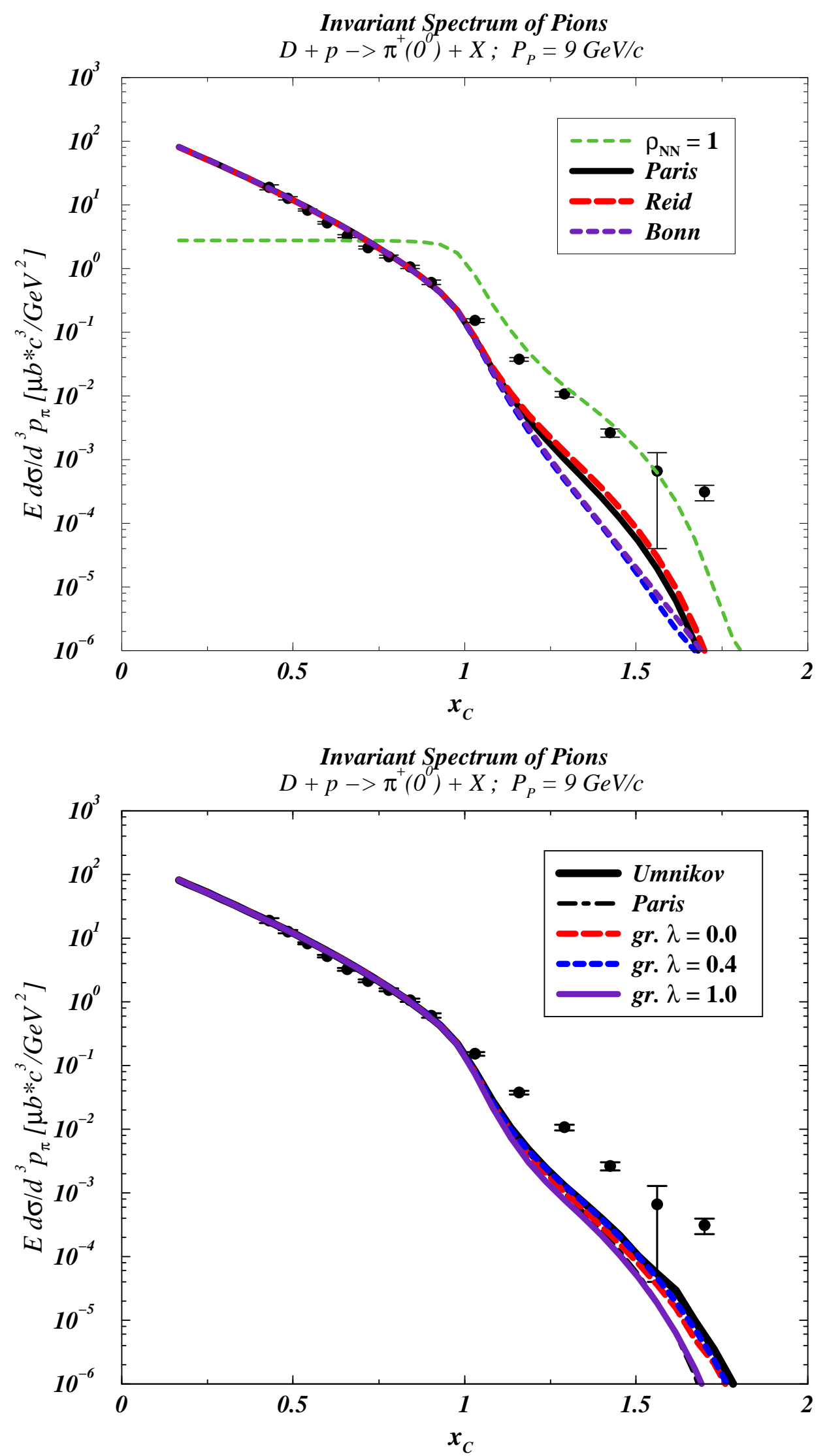

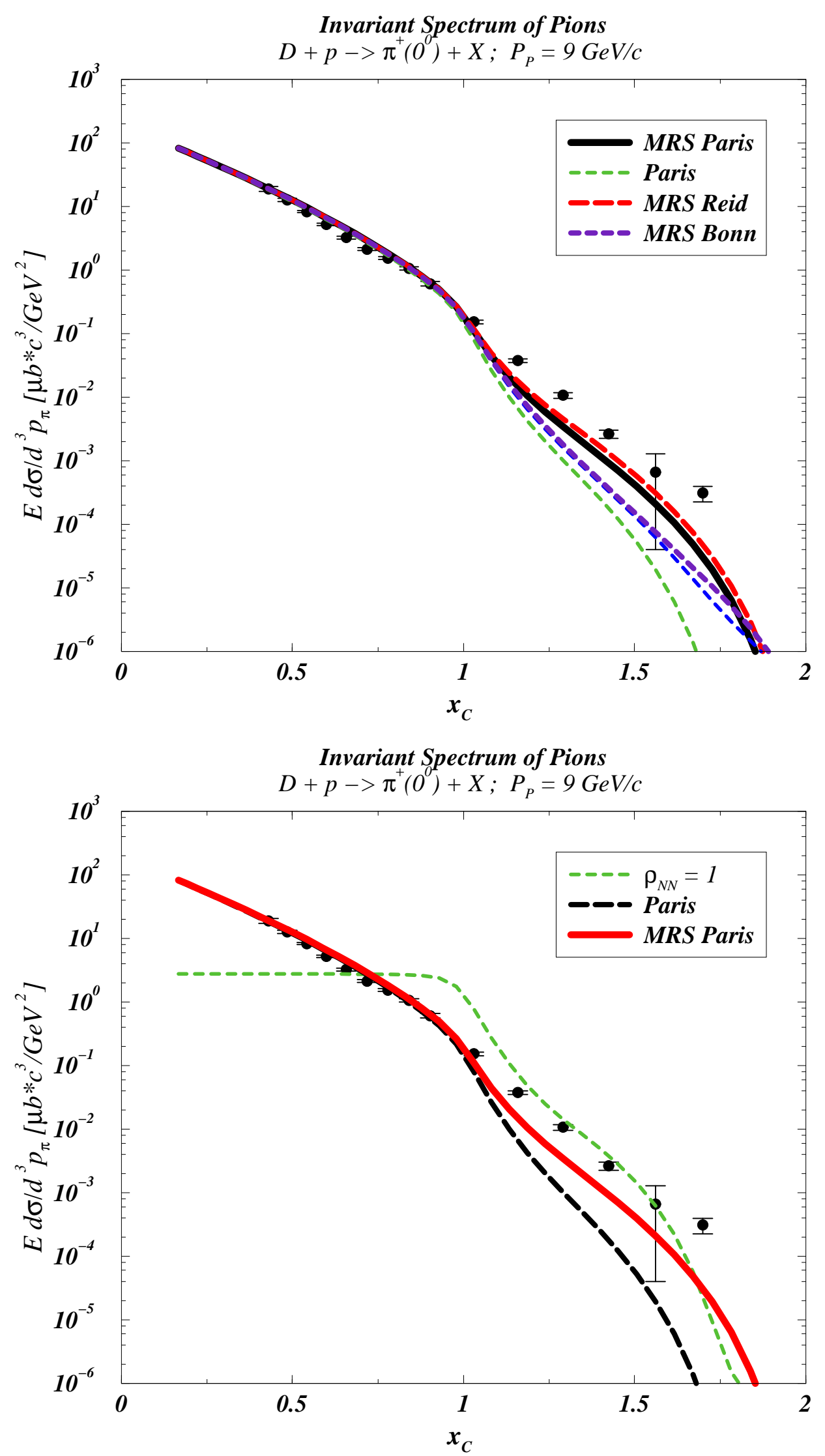

\section{Prague'2000}




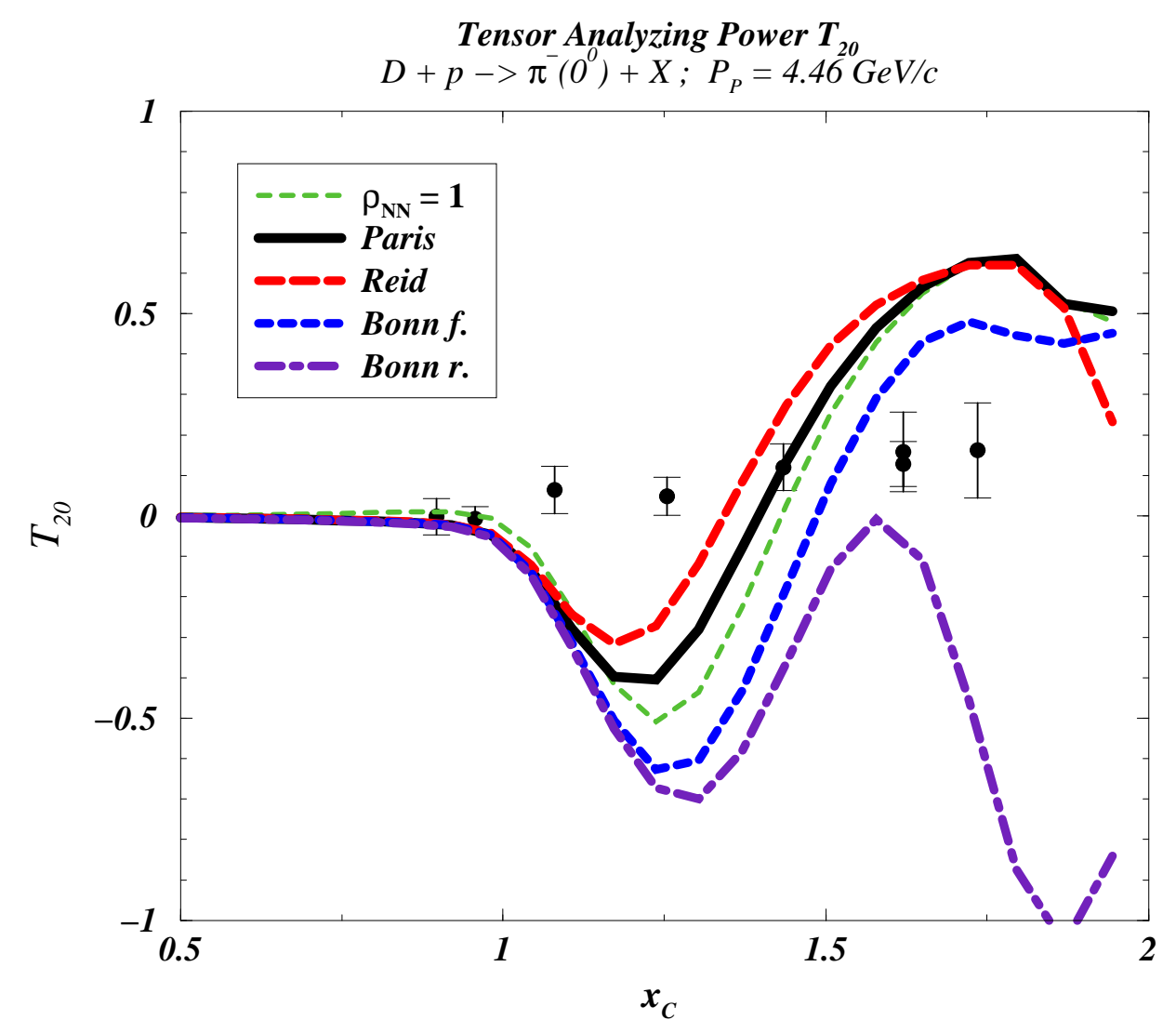

Tensor Analyzing Power $\boldsymbol{T}_{20}$ $D+p \rightarrow \pi^{-}\left(0^{\circ}\right)+X ; P_{P}=4.46 \mathrm{GeV} / \mathrm{c}$

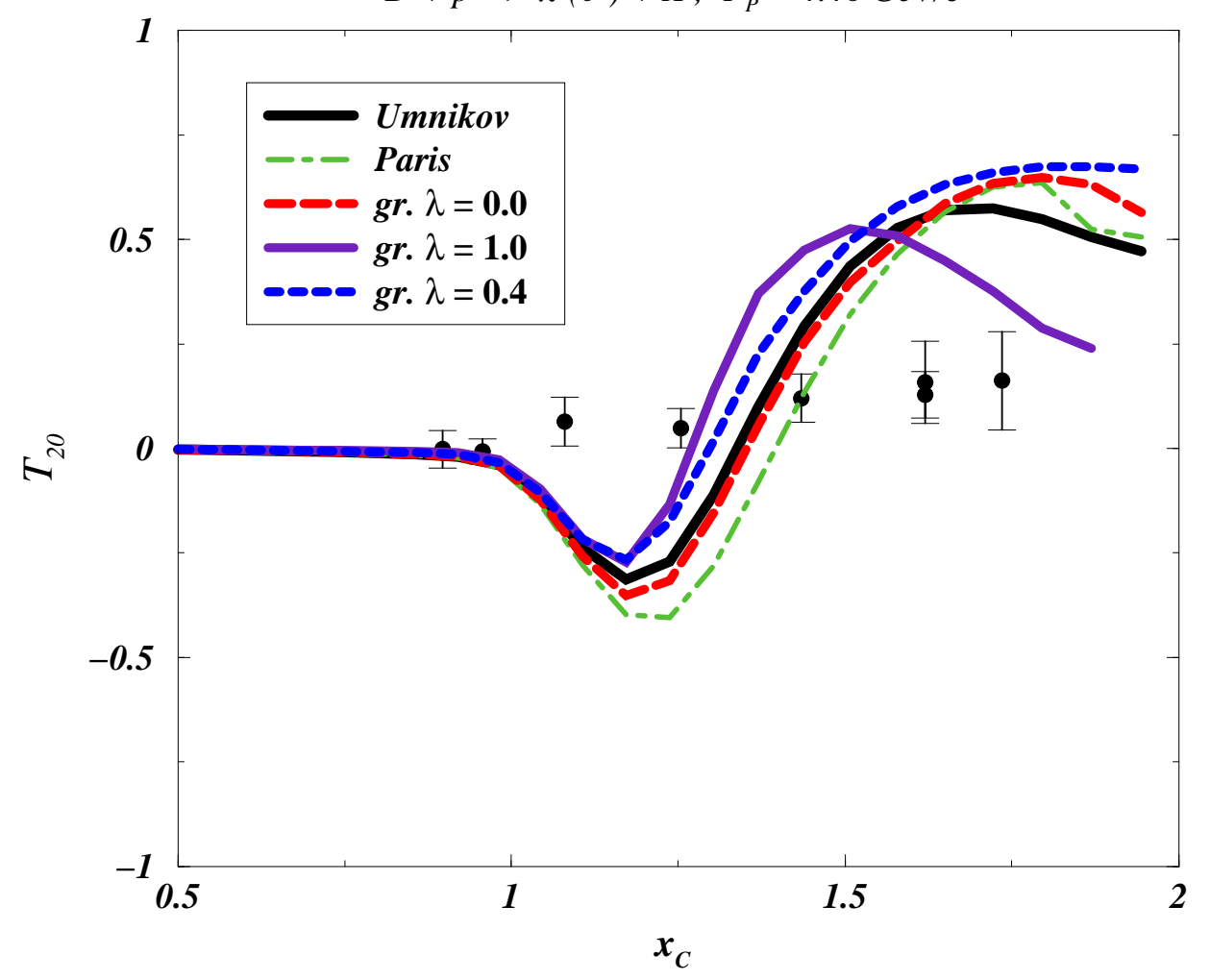




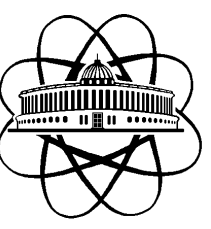

Tensor Analyzing Power $\boldsymbol{T}_{20}$

$D+p->\pi^{-}\left(0^{\circ}\right)+X ; P_{P}=4.46 \mathrm{GeV} / \mathrm{c}$

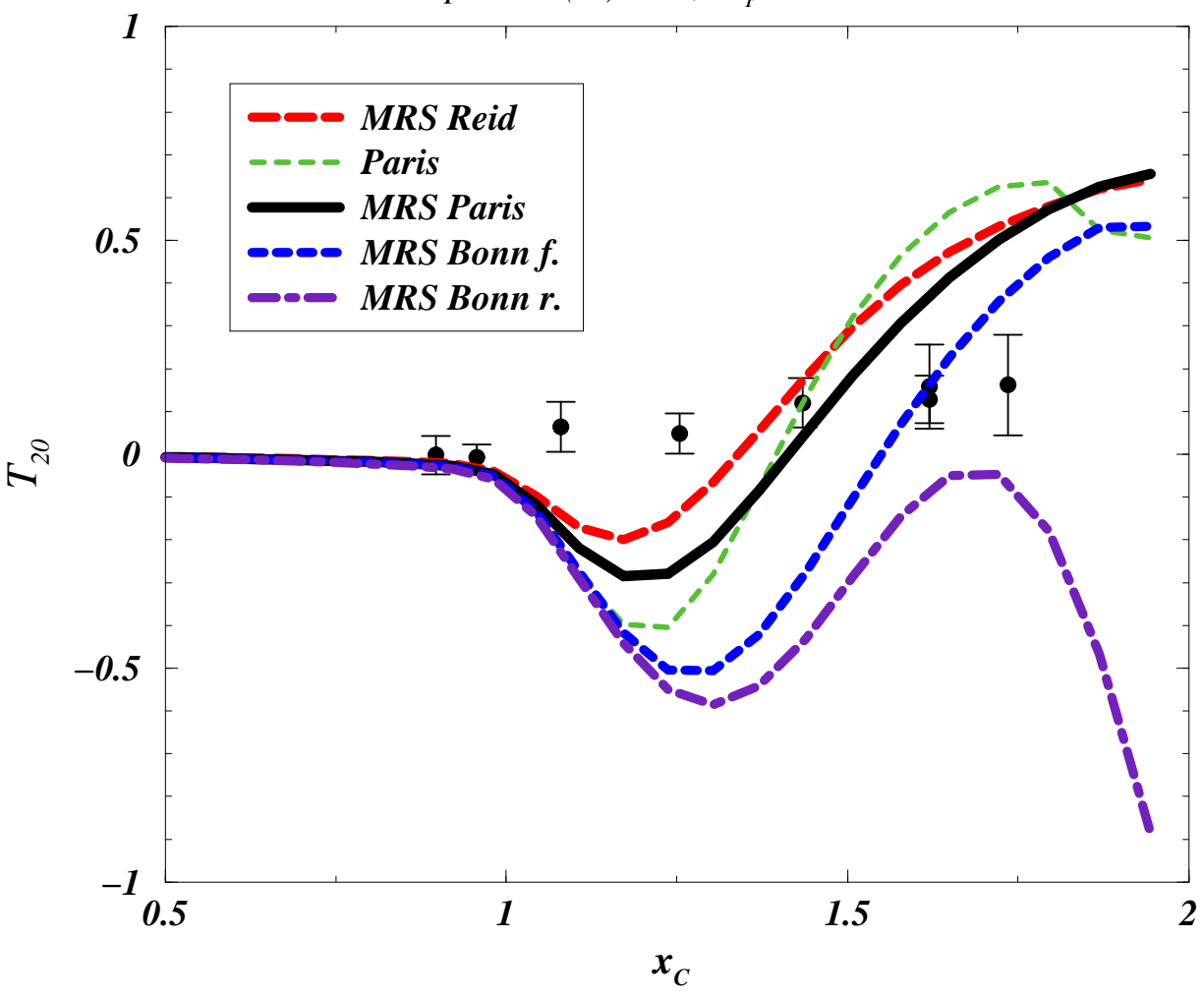

Tensor Analyzing Power $\boldsymbol{T}_{20}$

$D+p->\pi^{-}\left(0^{\circ}\right)+X ; P_{P}=4.46 \mathrm{GeV} / \mathrm{c}$

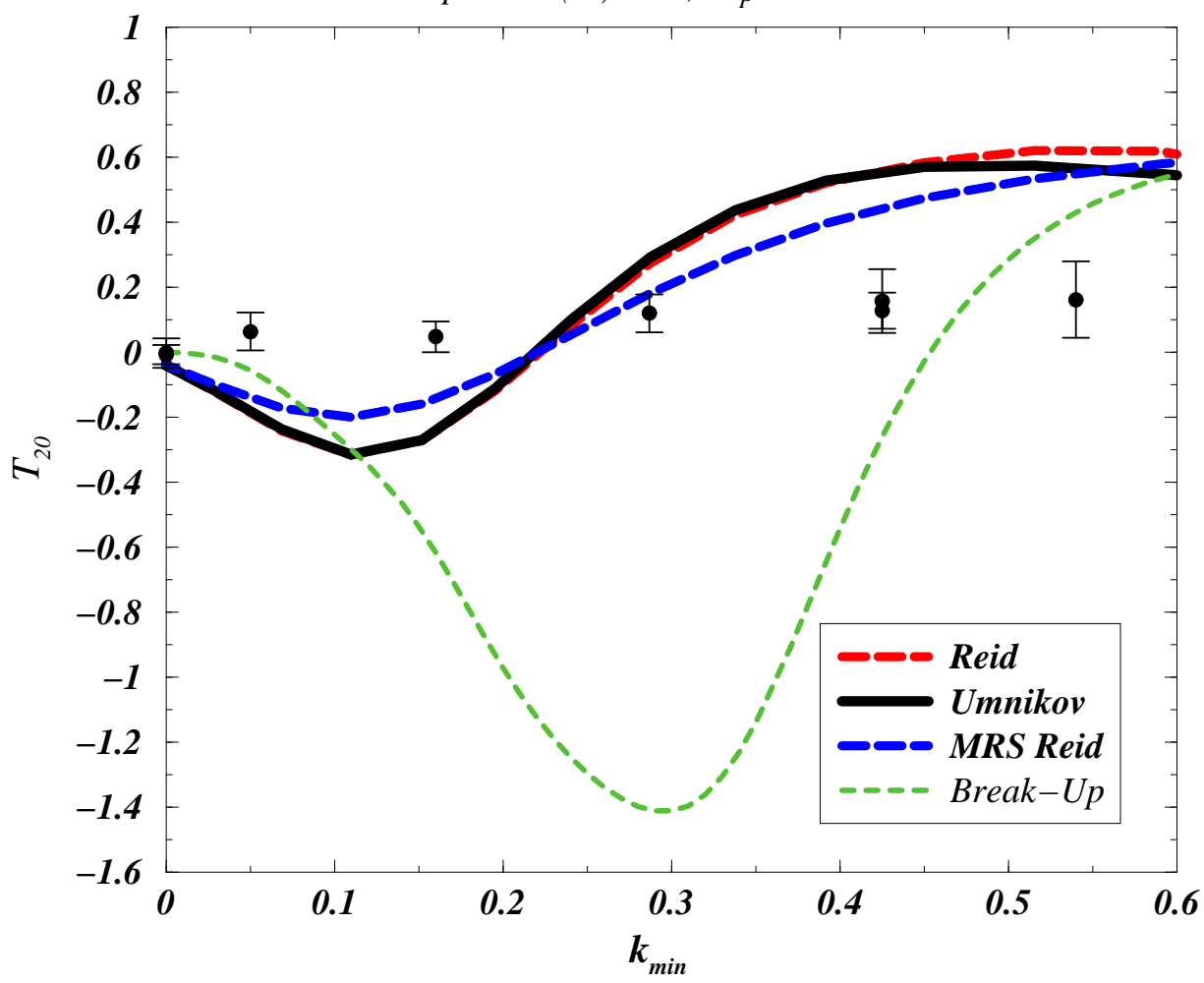

\section{Prague'2000}




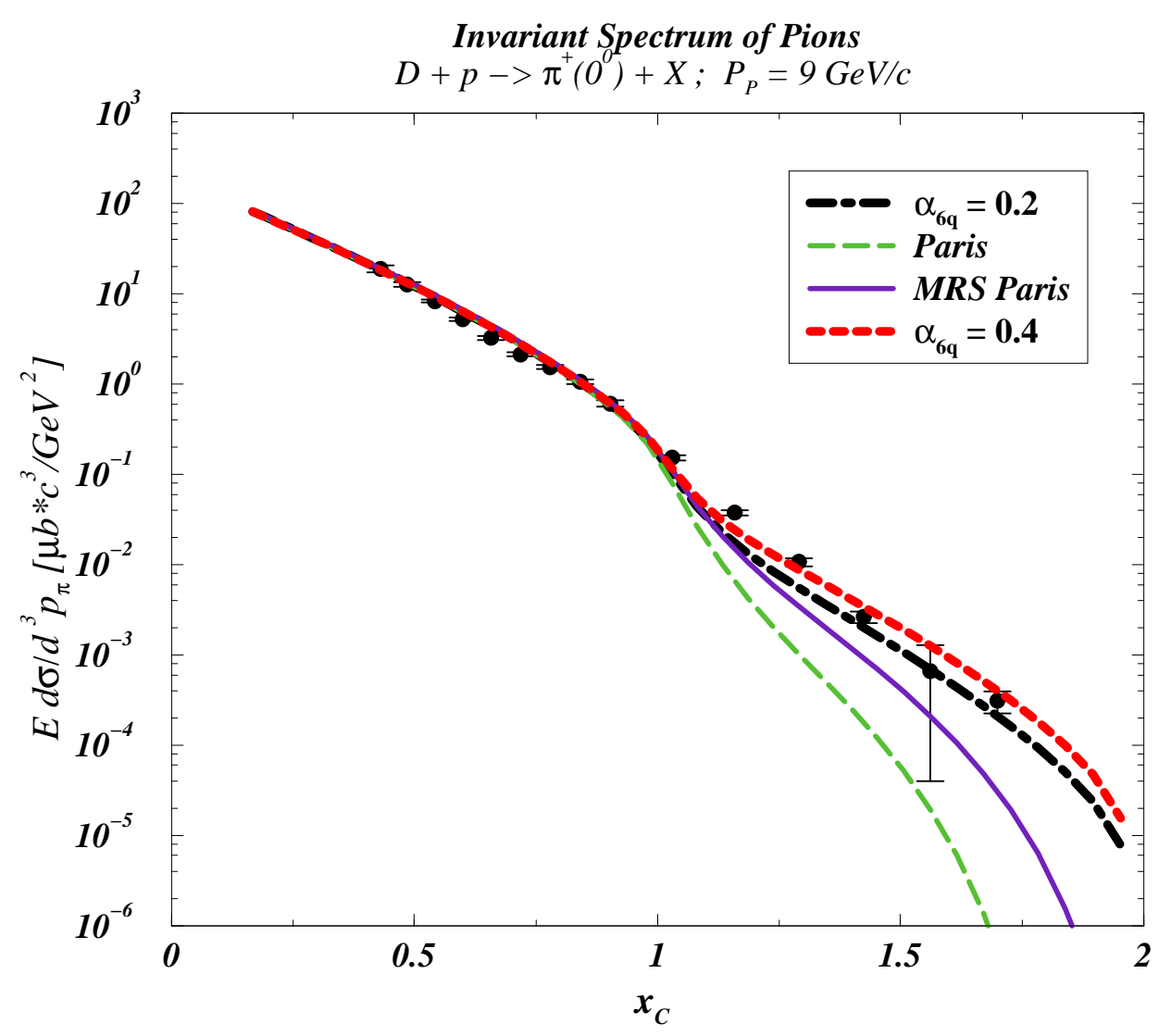

The parameter $\alpha_{6 q}$ is the probability for the non-nucleon component in the deuteron which is a state of two colorless $(3 q)$ systems. 


\section{Physical results and conclusions}

I. The polarization observables are very sensitive to the kind of DWF, especialy at $x_{\mathcal{C}}>1$.

II. The inclusion of $P$-wave in DWF doesn't result in the description of the inclusive spectrum and $T_{20}$ at $x_{\mathcal{C}}>1$.

III. The inclusive spectrum of pions is very sensitive to $\rho_{N N \rightarrow \pi X}$ and to change:

$$
\boldsymbol{q}^{2} \Longrightarrow \boldsymbol{k}^{2}=\frac{m^{2}+\boldsymbol{q}_{\perp}^{2}}{4 x(1-x)}-m^{2}
$$

in comparison to the one of $T_{20}$.

IV. The big uncertainty for $\rho_{p D}^{\pi}$ and small one for $T_{20}$.

V. Within the conventional nuclear physics $T_{20}$ can't be described at $x_{\mathcal{C}}>1$. 


\section{Summary and Outlook}

- It is quite incorrect to use a nonrelativistic DWF by the analysis of $D-N$ fragmentation to hadrons, in particular pions.

- The unique procedure for inclusion of relativistic effects in deuteron has not been yet found.

- The very interesting experimental data about $T_{20}$ showing approximately zero values at $x_{\mathcal{C}} \geq 1$ are not reproduced by theoretical calculus using even different kinds of the relativistic DWF what can indicate to a possible existence of non-nucleon degrees of freedom or principle new mechanism of pion production in the kinematical region forbidden for free $N-N$ scattering.

- $T_{20}$ by the fragmentation of deuterons to pions can't be described within this physics in the wide interval of $x_{\mathcal{C}}$, at $x_{\mathcal{C}} \geq 1$. It can be pointed out the more interest to study experimentally and theoretically namely the pion fragmentation of deuteron in order to investigate the deuteron structure at small distances.

\section{Prague'2000}

